\title{
On the joint analysis of the total discounted payments to policyholders and shareholders: threshold dividend strategy
}

\author{
Eric C.K. Cheung* \\ Department of Statistics and Actuarial Science, University of Hong Kong, Pokfulam, Hong Kong \\ Haibo Liu \\ Department of Statistics and Actuarial Science, University of lowa, lowa City, IA 52242, USA
}

\begin{abstract}
In insurance risk theory, dividend and aggregate claim amount are of great research interest as they represent the insurance company's payments to its shareholders and policyholders, respectively. Since the analyses of these two quantities are performed separately in the literature, the companion paper by Cheung et al. generalised the Gerber-Shiu expected discounted penalty function by further incorporating the moments of the aggregate discounted claims until ruin and the discounted dividends until ruin. While Cheung et al. considered the compound Poisson model with a dividend barrier in which ruin occurs almost surely, the present paper looks at this generalised Gerber-Shiu function under a threshold dividend strategy where the insurer has a positive survival probability. Because the Gerber-Shiu function is only defined for sample paths leading to ruin, we will additionally study the joint moments of the aggregate discounted claims and the discounted dividends without ruin occurring. Some explicit formulas are derived when the individual claim distribution follows a combination of exponentials. Numerical illustrations involving the correlation between aggregate discounted claims and discounted dividends are given. For the case where ruin occurs, we additionally compute the correlations between the time of ruin and the above two quantities.
\end{abstract}

\section{Keywords}

Compound Poisson risk model; Threshold dividend strategy; Aggregate discounted claims; Discounted dividends; Correlation.

\section{Introduction}

In the classical compound Poisson insurance risk model, the baseline (i.e., without dividends) surplus process $\{U(t)\}_{t \geq 0}$ of the insurer is modelled as

$$
U(t)=u+c t-S(t), \quad t \geq 0
$$

where $u=U(0) \geq 0$ is the initial surplus, $c>0$ the incoming premium rate per unit time, and $\{S(t)\}_{t \geq 0}$ the aggregate claims process. Specifically, the aggregate claim amount until time $t$ is given by $S(t)=\sum_{k=1}^{N(t)} Y_{k}$, where $\{N(t)\}_{t \geq 0}$ is a Poisson process with rate $\lambda>0$, and $\left\{Y_{k}\right\}_{k=1}^{\infty}$ a sequence of independent and identically distributed positive continuous random variables representing the

*Correspondence to: Eric C.K. Cheung, Department of Statistics and Actuarial Science, University of Hong Kong, Pokfulam, Hong Kong. Tel: (852) 3917-8315; Fax: (852) 2858-9041; E-mail: eckc@hku.hk 
individual claim amounts. Moreover, it is assumed that $\{N(t)\}_{t \geq 0}$ and $\left\{Y_{k}\right\}_{k=1}^{\infty}$ are independent. For later use, the common probability density function of the random variables $\left\{Y_{k}\right\}_{k=1}^{\infty}$ is denoted by $p(\cdot)$ and its Laplace transform is $\tilde{p}(s)=\int_{0}^{\infty} e^{-s y} p(y) d y$.

The seminal paper by de Finetti (1957) suggested that the insurer should redistribute some of its surplus to its shareholders, leading to extensive study of dividend strategies in various insurance risk models (see e.g., Albrecher \& Thonhauser, 2009; Avanzi, 2009 for reviews). The most commonly studied dividend strategy in the literature is the barrier strategy (e.g., Gerber, 1979) in which any excess of the surplus over a fixed barrier is immediately paid to the shareholders as dividends. Although such a strategy is optimal as far as the maximisation of the expected discounted dividends until ruin is concerned when $p(\cdot)$ is completely monotone (e.g., Loeffen, 2008, theorem 3), it results in an ultimate ruin probability of one which is practically undesirable. In this paper, we shall impose a threshold dividend strategy (e.g., Gerber \& Shiu, 2006; Lin \& Pavlova, 2006) to the surplus process (1.1), so that part of the incoming premium rate is paid as dividends whenever the insurer's surplus exceeds a fixed threshold level $b>0$. Denoting the dividend rate by $\alpha>0$ and the premium rate by $c_{1}=c$, the net premium rate is $c_{2}=c_{1}-\alpha$ when the surplus is above $b$. Therefore, the modified risk process $\left\{U_{b}(t)\right\}_{t \geq 0}$ under the above threshold strategy follows the dynamics:

$$
d U_{b}(t)= \begin{cases}c_{1} d t-d S(t), & U_{b}(t)<b \\ c_{2} d t-d S(t), & U_{b}(t) \geq b\end{cases}
$$

and the initial surplus is given by $u=U_{b}(0) \geq 0$. The time of ruin of $\left\{U_{b}(t)\right\}_{t \geq 0}$ is defined to be $\tau_{b}=\inf \left\{t \geq 0: U_{b}(t)<0\right\}$ with the convention that $\tau_{b}=\inf \emptyset=\infty$ if $U_{b}(t) \geq 0$ for all $t \geq 0$. Then, the ruin probability is given by $\psi(u ; b)=\operatorname{Pr}\left\{\tau_{b}<\infty \mid U_{b}(0)=u\right\}$. The positive security loading condition $c_{2}>\lambda E\left[Y_{1}\right]$ is assumed to ensure that $\psi(u ; b)<1$ for all $u \geq 0$ (e.g., Kyprianou, 2013, corollary 8.5). Note that $D(t)=U(t)-U_{b}(t)$ is the total dividends paid until time $t$. An important quantity of interest is the total discounted dividends until ruin, as it represents the value of firm in corporate finance. In the present context, it is defined by

$$
D_{\delta}\left(\tau_{b}\right)=\int_{0}^{\tau_{b}} e^{-\delta s} d D(s)=\alpha \int_{0}^{\tau_{b}} e^{-\delta s} 1_{\left\{U_{b}(s) \geq b\right\}} d s
$$

where $\delta>0$ is the force of interest and $1_{A}$ the indicator function of the event $A$. When each claim amount $Y_{k}$ is exponentially distributed, Gerber \& Shiu (2006, section 9) showed that the threshold strategy is optimal in maximising the expected discounted dividends until ruin for restricted dividend rate. Given a threshold strategy, Dickson \& Drekic (2006) analysed the optimal pair of threshold level and dividend rate that maximises the expectation of $D_{\delta}\left(\tau_{b}\right)$ under a ruin probability constraint, whereas Cheung et al. (2008) derived the higher moments of $D_{\delta}\left(\tau_{b}\right)$ and computed the optimal threshold minimising the coefficient of variation of $D_{\delta}\left(\tau_{b}\right)$.

Apart from $D_{\delta}\left(\tau_{b}\right)$, which is the total discounted payment made by the insurance company to its shareholders, another quantity of interest is the aggregate discounted claim amount payable to the policyholders until ruin, namely $\int_{0}^{\tau_{b}} e^{-\delta t} d S(t)$, where $\delta>0$ is the force of interest. More generally, one may consider the aggregate discounted claim costs until ruin defined by

$$
Z_{\delta}\left(\tau_{b}\right)=\sum_{k=1}^{N\left(\tau_{b}\right)} e^{-\delta T_{k}} f\left(Y_{k}\right)
$$

where $T_{k}$ is the time of the $k$ th claim (which is the $k$ th arrival time of the Poisson process $\left.\{N(t)\}_{t \geq 0}\right)$, and $f(\cdot)$ is a non-negative "cost function" applied to each claim. Clearly, if $f(y)=y$ then $Z_{\delta}\left(\tau_{b}\right)$ 
becomes $\sum_{k=1}^{N\left(\tau_{b}\right)} e^{-\delta T_{k}} Y_{k}=\int_{0}^{\tau_{b}} e^{-\delta t} d S(t)$. We remark that the aggregate discounted claim amount here is different from the one considered by e.g. Taylor (1979), Willmot (1989), Léveillé \& Garrido (2001), and Woo \& Cheung (2013), which is concerned with the aggregate until a fixed time $t$ instead of the ruin time. The quantity $Z_{\delta}\left(\tau_{b}\right)$ in (1.3) has gained some attention in recent years (in models without dividends, i.e., $\alpha=0$ ). For example, the expectation of $Z_{\delta}\left(\tau_{b}\right)$ was studied by Cai et al. (2009, section 6) and Feng (2009a, section 4.2, 2009b, section 5.2) in the compound Poisson and phase-type renewal risk models, whereas Cheung \& Feng (2013) analysed the higher moments of $Z_{\delta}\left(\tau_{b}\right)$ in a Markovian arrival process.

In addition to the discounted dividends, the Gerber-Shiu expected discounted penalty function has also been widely studied in insurance risk theory since the seminal paper by Gerber \& Shiu (1998) was published. In the present model, it is defined by

$$
\phi_{\delta}(u ; b)=E\left[e^{-\delta \tau_{b}} w\left(U_{b}\left(\tau_{b}^{-}\right),\left|U_{b}\left(\tau_{b}\right)\right|\right) 1_{\left\{\tau_{b}<\infty\right\}} \mid U_{b}(0)=u\right], \quad u \geq 0
$$

where $\delta \geq 0$ can be regarded as the force of interest or the Laplace transform argument with respect to the time of ruin $\tau_{b}$, and $w(\cdot, \cdot)$ is a non-negative "penalty" as a function of the surplus immediately before ruin $U_{b}\left(\tau_{b}^{-}\right)$and the deficit at ruin $\left|U_{b}\left(\tau_{b}\right)\right|$. Typically, $w(\cdot, \cdot)$ is assumed to satisfy some mild integrability conditions. While the Gerber-Shiu function (1.4) was studied by Lin \& Pavlova (2006), some related results on the corresponding discounted densities were given by Zhou (2004, section 4). Interested readers are referred to e.g. Albrecher et al. (2007, section 2), Badescu et al. (2007a), Zhu \& Yang (2008), Lu \& Li (2009), and Kyprianou \& Loeffen (2010) for the analysis of the discounted dividends and the Gerber-Shiu function in more general processes such as the generalised $\operatorname{Erlang}(n)$ renewal model, risk model with Markovian claim arrivals, and the Lévy insurance risk process. We also remark that a more general multi-threshold dividend strategy was also considered by e.g. Albrecher \& Hartinger (2007), Badescu et al. (2007b), and Lin \& Sendova (2008).

In almost all works in the literature, the analyses of the discounted dividends (1.2), the aggregate discounted claim costs (1.3) and (the random variables in) the Gerber-Shiu function (1.4) were performed separately. Therefore, Cheung et al. (2015) proposed an extended version of the Gerber-Shiu function defined as

$$
\begin{aligned}
\phi_{\delta_{1}, \delta_{2}, \delta_{3}, n, m}(u ; b) & =\phi_{\delta_{123}, n, m}(u ; b) \\
& =E\left[e^{-\delta_{1} \tau_{b}} D_{\delta_{2}}^{n}\left(\tau_{b}\right) Z_{\delta_{3}}^{m}\left(\tau_{b}\right) w\left(U_{b}\left(\tau_{b}^{-}\right),\left|U_{b}\left(\tau_{b}\right)\right|\right) 1_{\left\{\tau_{b}<\infty\right\}} \mid U_{b}(0)=u\right], \quad u \geq 0
\end{aligned}
$$

where $n, m \in \mathbb{N}$ (with $\mathbb{N}$ being the set of non-negative integers) are the orders of moments of $D_{\delta_{2}}\left(\tau_{b}\right)$ and $Z_{\delta_{3}}\left(\tau_{b}\right)$. It is assumed that the cost function $f(\cdot)$ satisfies some mild integrability conditions (see Lemmas 2 and 3). Moreover, we assume $\delta_{1} \geq 0$, while $\delta_{2}, \delta_{3}>0$ are possibly different forces of interest used to discount dividends and claims for the shareholders and policyholders, respectively. Note that the indicator function $1_{\left\{\tau_{b}<\infty\right\}}$ does not appear in Cheung et al.'s (2015) definition since they considered a dividend barrier strategy for which ruin occurs almost surely (a.s.). For notational convenience, we shall use the abbreviation $\phi_{\delta_{123}, n, m}(u ; b)$ for $\phi_{\delta_{1}, \delta_{2}, \delta_{3}, n, m}(u ; b)$ when it does not cause any confusion. Obviously, if $n=m=0$ then $\phi_{\delta_{123}, 0,0}(u ; b)=\phi_{\delta_{1}}(u ; b)$ reduces to the classical Gerber-Shiu function defined in (1.4). Under a compound Poisson risk model with a dividend barrier, Cheung et al. (2015) applied $\phi_{\delta_{123}, n, m}(u ; b)$ to find various covariance measures between ruin-related quantities such as the discounted dividends until ruin and the aggregate discounted claims until ruin. Through some numerical examples, they demonstrated that the covariance between the above two random variables may take positive or negative value and gave some interpretations as well. The motivation for calculating the above covariance (or the resulting correlation) is 
as follows. Because the payments to the policyholders (claims) and the shareholders (dividends) both come from the same source, namely the insurer's surplus, it is interesting to see whether the discounted dividends and the aggregate discounted claims tend to move in the same or opposite direction. A high positive correlation indicates that the needs of the two groups could indeed be satisfied at the same time, but a negative correlation may suggest conflicting interests between the two groups. We remark that Gerber-Shiu-type functions resembling (1.5) were also introduced and analysed by Cheung (2013) and Cheung \& Woo (2016) in the absence of dividends. While the former contribution considered (1.5) where $n=0, \delta_{1}=k \delta_{3}$ for some $k \in \mathbb{N}$ and the penalty $w$ only depends on the deficit in renewal risk models with general interclaim times and exponential claims, the latter looked at (1.5), where $n=0$ and $w$ further depends on the surplus immediately after the second last claim before ruin in the dependent Sparre Andersen risk model. The latter work was also extended to a discrete time framework by Woo \& Liu (2014).

It is instructive to note that the Gerber-Shiu function (1.5) only takes into account the sample paths of $\left\{U_{b}(t)\right\}_{t \geq 0}$ for which ruin occurs. Under the loading condition $c_{2}>\lambda E\left[Y_{1}\right]$, the process $\left\{U_{b}(t)\right\}_{t \geq 0}$ has a positive survival probability. For these sample paths where $\left\{U_{b}(t)\right\}_{t \geq 0}$ survives forever (i.e., $\tau_{b}=\infty$ ), the discounted dividends $D_{\delta_{2}}\left(\tau_{b}\right)$ and the aggregate discounted claim costs $Z_{\delta_{3}}\left(\tau_{b}\right)$ are still defined although $U_{b}\left(\tau_{b}^{-}\right)$and $\left|U_{b}\left(\tau_{b}\right)\right|$ are not. Therefore, we will also analyse the joint moments of $D_{\delta_{2}}\left(\tau_{b}\right)$ and $Z_{\delta_{3}}\left(\tau_{b}\right)$ without ruin occurring via

$$
\varphi_{\delta_{2}, \delta_{3}, n, m}(u ; b)=\varphi_{\delta_{23}, n, m}(u ; b)=E\left[D_{\delta_{2}}^{n}\left(\tau_{b}\right) Z_{\delta_{3}}^{m}\left(\tau_{b}\right) 1_{\left\{\tau_{b}=\infty\right\}} \mid U_{b}(0)=u\right], \quad u \geq 0
$$

where $n, m \in \mathbb{N}$ and $\delta_{2}, \delta_{3}>0$. Note that the usual joint moments for all sample paths can readily be obtained as

$$
E\left[D_{\delta_{2}}^{n}\left(\tau_{b}\right) Z_{\delta_{3}}^{m}\left(\tau_{b}\right) \mid U_{b}(0)=u\right]=\left.\phi_{\delta_{123, n, m}}(u ; b)\right|_{\delta_{1}=0, w \equiv 1}+\varphi_{\delta_{23}, n, m}(u ; b)
$$

This paper is organised as follows. In section 2, the integro-differential equations (IDEs) for $\phi_{\delta_{123}, n, m}(u ; b)$ and $\varphi_{\delta_{23}, n, m}(u ; b)$ as well as the corresponding continuity conditions and limiting behaviours as $u \rightarrow \infty$ are given. Under the assumption that each individual claim is distributed as a combination of exponentials, section 3 provides some explicit expressions for $\phi_{\delta_{123}, n, m}(u ; b)$ and $\varphi_{\delta_{23}, n, m}(u ; b)$ when $f(y)=y$ and $w(x, y)$ depends on the deficit argument $y$ but not $x$. Because the derivation of the IDEs and the procedure towards the exact solutions are quite standard but require tedious and careful calculations, only the main results are stated in sections 2 and 3 with the details of the proofs provided in the Appendix. Section 4 is concerned with some numerical illustrations in which we compute the correlation between the discounted dividends and the aggregate discounted claims separately for the cases of ruin and survival. For the case where ruin occurs, the correlations between the time of ruin and the above two quantities are also given. Probabilistic interpretations follow as well. Section 5 ends the paper with some concluding remarks.

\section{General Results}

Due to the presence of the dividend threshold $b$, the IDEs in $u$ satisfied by $\phi_{\delta_{123}, n, m}(u ; b)$ are different depending on whether $0 \leq u<b$ or $u \geq b$ (and hence the solution forms will also be different as in section $3)$. Therefore, we shall denote $\phi_{\delta_{123}, n, m}(u ; b)$ by $\phi_{L, \delta_{123}, n, m}(u ; b)$ for $0 \leq u<b$ and $\phi_{U, \delta_{123}, n, m}(u ; b)$ for $u \geq b$, where " $L$ " and " $U$ " stand for "Lower" and "Upper" layers, respectively. Furthermore, we shall

use $\phi_{\delta_{123}, n, m}^{\prime}(u ; b)=(d / d u) \phi_{\delta_{123}, n, m}(u ; b)$ to denote the derivative of $\phi_{\delta_{123}, n, m}(u ; b)$ with respect to the first argument $u$. Similar notations will be applied to $\varphi_{\delta_{23}, n, m}(u ; b)$ and other related functions as well. The proofs of the theorems and lemmas in this section are given in the Appendix. 


\subsection{IDEs and continuity condition for $\phi_{\delta_{123}, n, m}(u ; b)$ when $n=0$}

First, we consider $\phi_{\delta_{123}, 0, m}(u ; b)$ (i.e., $\left.n=0\right)$ so that the dividend component $D_{\delta_{2}}\left(\tau_{b}\right)$ does not appear in the definition (1.5). For notational convenience, we write

$$
\phi_{\delta_{13}, m}(u ; b)=\phi_{\delta_{123}, 0, m}(u ; b)=E\left[e^{-\delta_{1} \tau_{b}} Z_{\delta_{3}}^{m}\left(\tau_{b}\right) w\left(U_{b}\left(\tau_{b}^{-}\right), \mid U_{b}\left(\tau_{b}\right)\right) 1_{\left\{\tau_{b}<\infty\right\}} \mid U_{b}(0)=u\right], \quad u \geq 0
$$

The IDEs and continuity condition for $\phi_{\delta_{13}, m}(u ; b)$ are stated in the following theorem, where $\phi_{\delta_{13}, m}(u ; b)=\phi_{L, \delta_{13}, m}(u ; b)$ for $0 \leq u<b$ and $\phi_{\delta_{13}, m}(u ; b)=\phi_{U, \delta_{13}, m}(u ; b)$ for $u \geq b$ according to our afore-mentioned convention.

Theorem 1 For $m \in \mathbb{N}$, the Gerber-Shiu function $\phi_{\delta_{13}, m}(u ; b)$ in (2.1) satisfies the IDEs, for $0<u<b$

$$
\begin{aligned}
& c_{1} \phi_{L, \delta_{13}, m}^{\prime}(u ; b)-\left(\lambda+\delta_{1}+m \delta_{3}\right) \phi_{L, \delta_{13}, m}(u ; b)+\lambda \sum_{i=0}^{m}\left(\begin{array}{c}
m \\
i
\end{array}\right) \int_{0}^{u} f^{m-i}(y) \phi_{L, \delta_{13}, i}(u-y ; b) p(y) d y \\
& \quad+\lambda \int_{u}^{\infty} f^{m}(y) w(u, y-u) p(y) d y=0
\end{aligned}
$$

and for $u>b$

$$
\begin{aligned}
& c_{2} \phi_{U, \delta_{13}, m}^{\prime}(u ; b)-\left(\lambda+\delta_{1}+m \delta_{3}\right) \phi_{U, \delta_{13}, m}(u ; b)+\lambda \sum_{i=0}^{m}\left(\begin{array}{c}
m \\
i
\end{array}\right) \int_{0}^{u-b} f^{m-i}(y) \phi_{U, \delta_{13, i}}(u-y ; b) p(y) d y \\
& \quad+\lambda \sum_{i=0}^{m}\left(\begin{array}{c}
m \\
i
\end{array}\right) \int_{u-b}^{u} f^{m-i}(y) \phi_{L, \delta_{13}, i}(u-y ; b) p(y) d y+\lambda \int_{u}^{\infty} f^{m}(y) w(u, y-u) p(y) d y=0
\end{aligned}
$$

In addition, $\phi_{\delta_{13}, m}(u ; b)$ is continuous at $u=b$, i.e.

$$
\phi_{L, \delta_{13}, m}\left(b^{-} ; b\right)=\phi_{U, \delta_{13}, m}(b+; b)
$$

Remark 1 As $\phi_{\delta_{13}, m}(u ; b)$ reduces to the classical Gerber-Shiu function $\phi_{\delta_{1}}(u ; b)$ when $m=0$, it is noted that the results in Lin \& Pavlova (2006, theorem 3.1) can be retrieved from the above theorem by putting $m=0$. Note also that the determination of $\phi_{\delta_{13}, m}(u ; b)$ is recursive in $m$, with the starting point given by $\phi_{\delta_{1}}(u ; b)$. Assuming that the lower-order Gerber-Shiu functions $\phi_{\delta_{13}, i}(\cdot ; b)$ for $i=0$, $1, \ldots, m-1$ are known, it is observed that the IDE (2.3) involves both $\phi_{L, \delta_{13}, m}(\cdot ; b)$ in the lower layer and $\phi_{U, \delta_{13}, m}(\cdot ; b)$ in the upper layer as unknown functions, while $(2.2)$ only involves $\phi_{L, \delta_{13}, m}(\cdot ; b)$. Therefore, the typical procedure is to first utilise (2.2) to determine the solution form of $\phi_{L, \delta_{13}, m}(\cdot ; b)$, and then attempt to find $\phi_{U, \delta_{13}, m}(\cdot ; b)$ in $(2.3)$ by treating $\phi_{L, \delta_{13}, m}(\cdot ; b)$ as known (see the proofs of theorems in section 3).

Remark 2 Having established the continuity of $\phi_{\delta_{13}, i}(\cdot ; b)$ for $i \in \mathbb{N}$ in the proof of Theorem 1, we observe from (2.2) that for each $m \in \mathbb{N}$ the derivative $\phi_{L, \delta_{13}, m}^{\prime}(u ; b)$ is continuous in $u$ in the layer $0<u<b$ if $\int_{u}^{\infty} f^{m}(y) w(u, y-u) p(y) d y$ is continuous in $u$. A sufficient condition for $\int_{u}^{\infty} f^{m}(y) w(u, y-u) p(y) d y$ to be continuous in $u$ is that the penalty $w(\cdot, \cdot)$ is a continuous function. For the same reason, $\phi_{U, \delta_{13}, m}^{\prime}(u ; b)$ is continuous in $u$ for $u>b$ under the same sufficient condition. However, although $\phi_{\delta_{13}, m}(u ; b)$ is continuous at $u=b$, the derivative $\phi_{\delta_{13}, m}^{\prime}(u ; b)$ is generally not continuous at $u=b$. To see this, letting $u \rightarrow b^{-}$in (2.2) and $u \rightarrow b^{+}$in (2.3) (assuming $\int_{u}^{\infty} f^{m}(y) w(u, y-u) p(y) d y$ is continuous at $\left.u=b\right)$ and comparing the two equations gives rise to

$$
c_{1} \phi_{L, \delta_{13}, m}^{\prime}\left(b^{-} ; b\right)=c_{2} \phi_{U, \delta_{13}, m}^{\prime}\left(b^{+} ; b\right)
$$


which generalises the result at the end of section 3 in Lin \& Pavlova (2006) (see also Gerber \& Shiu, 2006, equation (10.5)). Therefore, $\phi_{L, \delta_{13}, m}^{\prime}\left(b^{-} ; b\right) \neq \phi_{U, \delta_{13}, m}^{\prime}\left(b^{+} ; b\right)$ unless $c_{1}=c_{2} \quad$ (or equivalently $\alpha=0$ ).

\subsection{IDEs and continuity condition for $\phi_{\delta_{123}, n, m}(u ; b)$ when $n \in \mathbb{N}^{+}$}

Next, we look at the Gerber-Shiu function $\phi_{\delta_{123}, n, m}(u ; b)$ defined in $(1.5)$ when $n \in \mathbb{N}^{+}$and $m \in \mathbb{N}$ (where $\mathbb{N}^{+}$is the set of positive integers). The following theorem gives the associated IDEs and continuity condition.

Theorem 2 For $n \in \mathbb{N}^{+}$and $m \in \mathbb{N}$, the Gerber-Shiu function $\phi_{\delta_{123}, n, m}(u ; b)$ in (1.5) satisfies the IDEs, for $0<u<b$

$$
\begin{aligned}
& c_{1} \phi_{L, \delta_{123}, n, m}^{\prime}(u ; b)-\left(\lambda+\delta_{1}+n \delta_{2}+m \delta_{3}\right) \phi_{L, \delta_{123}, n, m}(u ; b) \\
& \quad+\lambda \sum_{i=0}^{m}\left(\begin{array}{c}
m \\
i
\end{array}\right) \int_{0}^{u} f^{m-i}(y) \phi_{L, \delta_{123}, n, i}(u-y ; b) p(y) d y=0
\end{aligned}
$$

and for $u>b$

$$
\begin{aligned}
& c_{2} \phi_{U, \delta_{123}, n, m}^{\prime}(u ; b)-\left(\lambda+\delta_{1}+n \delta_{2}+m \delta_{3}\right) \phi_{U, \delta_{123}, n, m}(u ; b)+\alpha n \phi_{U, \delta_{123}, n-1, m}(u ; b) \\
& \quad+\lambda \sum_{i=0}^{m}\left(\begin{array}{c}
m \\
i
\end{array}\right) \int_{0}^{u-b} f^{m-i}(y) \phi_{U, \delta_{123}, n, i}(u-y ; b) p(y) d y \\
& \quad+\lambda \sum_{i=0}^{m}\left(\begin{array}{c}
m \\
i
\end{array}\right) \int_{u-b}^{u} f^{m-i}(y) \phi_{L, \delta_{123}, n, i}(u-y ; b) p(y) d y=0
\end{aligned}
$$

In addition, $\phi_{\delta_{123}, n, m}(u ; b)$ is continuous at $u=b$, i.e.

$$
\phi_{L, \delta_{123}, n, m}\left(b^{-} ; b\right)=\phi_{U, \delta_{123}, n, m}\left(b^{+} ; b\right)
$$

Remark 3 It is instructive to note from (2.5) and (2.6) that one requires a double recursion in both $n$ and $m$ to determine $\phi_{\delta_{123}, n, m}(u ; b)$ (see similar comments in Remark 1 concerning $\phi_{\delta_{13}, m}(u ; b)$ ). Furthermore, in parallel to Remark 2 (assuming $\int_{u}^{\infty} f^{m}(y) w(u, y-u) p(y) d y$ is continuous at $u=b$ ) it is clear that, for $n \in \mathbb{N}^{+}$

$$
c_{1} \phi_{L, \delta_{123}, n, m}^{\prime}\left(b^{-} ; b\right)=c_{2} \phi_{U, \delta_{123}, n, m}^{\prime}\left(b^{+} ; b\right)+\alpha n \phi_{U, \delta_{123}, n-1, m}(b ; b)
$$

i.e. the derivative of $\phi_{\delta_{123}, n, m}(u ; b)$ is not necessarily continuous at $u=b$.

Remark 4 Note that the IDEs (2.2) and (2.5) for $\phi_{\delta_{123}, n, m}(u ; b)$ in the lower layer are the same as those in theorems 1 and 2 in Cheung et al. (2015) concerning the dividend barrier strategy. This is because these IDEs are obtainable by considering an infinitesimal time interval, for which the dynamics of the surplus process are identical as no dividend is payable in the lower layer regardless of whether a barrier or a threshold strategy is implemented. Such an observation will allow us to reuse some of the intermediate results in Cheung et al. (2015) in section 3. 


\subsection{IDEs and continuity condition for $\varphi_{\delta_{23}, n, m}(u ; b)$}

In the next theorem, the IDEs and continuity condition concerning $\varphi_{\delta_{23}, n, m}(u ; b)$ (i.e., without ruin occurring) for $n, m \in \mathbb{N}$ will be provided. Because the dividend value $D_{\delta_{2}}\left(\tau_{b}\right)$ cannot be 0 on the set $\left\{\tau_{b}=\infty\right\}$, here we do not need to separate the analysis into the cases $n=0$ or $n \in \mathbb{N}^{+}$(unlike the Gerber-Shiu function $\phi_{\delta_{123}, n, m}(u ; b)$ in the previous two subsections).

Theorem 3 For $n, m \in \mathbb{N}$, the joint moment $\varphi_{\delta_{23}, n, m}(u ; b)$ in (1.6) satisfies the IDEs, for $0<u<b$ $c_{1} \varphi_{L, \delta_{23}, n, m}^{\prime}(u ; b)-\left(\lambda+n \delta_{2}+m \delta_{3}\right) \varphi_{L, \delta_{23}, n, m}(u ; b)+\lambda \sum_{i=0}^{m}\left(\begin{array}{c}m \\ i\end{array}\right) \int_{0}^{u} f^{m-i}(y) \varphi_{L, \delta_{23}, n, i}(u-y ; b) p(y) d y=0$

and for $u>b$

$$
\begin{aligned}
& c_{2} \varphi_{U, \delta_{23}, n, m}^{\prime}(u ; b)-\left(\lambda+n \delta_{2}+m \delta_{3}\right) \varphi_{U, \delta_{23}, n, m}(u ; b)+\alpha n \varphi_{U, \delta_{23}, n-1, m}(u ; b) \\
& \quad+\lambda \sum_{i=0}^{m}\left(\begin{array}{c}
m \\
i
\end{array}\right) \int_{0}^{u-b} f^{m-i}(y) \varphi_{U, \delta_{23}, n, i}(u-y ; b) p(y) d y \\
& \quad+\lambda \sum_{i=0}^{m}\left(\begin{array}{c}
m \\
i
\end{array}\right) \int_{u-b}^{u} f^{m-i}(y) \varphi_{L, \delta_{23}, n, i}(u-y ; b) p(y) d y=0
\end{aligned}
$$

In addition, $\varphi_{\delta_{23}, n, m}(u ; b)$ is continuous at $u=b$, i.e.

$$
\varphi_{L, \delta_{23}, n, m}\left(b^{-} ; b\right)=\varphi_{U, \delta_{23}, n, m}\left(b^{+} ; b\right)
$$

It is understood that $\varphi_{U, \delta_{23}, n-1, m}(u ; b)$ appearing in (2.9) is regarded as 0 when $n=0$.

Remark 5 For $n \in \mathbb{N}^{+}$and $m \in \mathbb{N}$, it is observed that the IDEs (2.5) and (2.6) in Theorem 2 satisfied by $\left.\phi_{\delta_{123}, n, m}(u ; b)\right|_{\delta_{1}=0}$ in the case of ruin are identical to the IDEs (2.8) and (2.9) in Theorem 3 for $\varphi_{\delta_{23}, n, m}(u ; b)$ concerning the case of survival. However, the full solutions to $\left.\phi_{\delta_{123}, n, m}(u ; b)\right|_{\delta_{1}=0}$ and $\varphi_{\delta_{23}, n, m}(u ; b)$ are generally different due to different limiting conditions as $u \rightarrow \infty$ (which will be discussed in the next subsection) and the fact that the lower-order moments appearing in the two sets of IDEs are different.

\subsection{Limits of $\phi_{\delta_{123}, n, m}(u ; b)$ and $\varphi_{\delta_{23}, n, m}(u ; b)$ as $u \rightarrow \infty$}

From Theorems 1 and 2, the Gerber-Shiu function $\phi_{\delta_{123}, n, m}(u ; b)$ satisfies two different IDEs in the lower and upper layers, and each IDE contains a derivative term. Therefore, the determination of the full solution of $\phi_{\delta_{123}, n, m}(u ; b)$ from the IDEs typically requires one more piece of information apart from the continuity condition. Similar comments are applicable to $\varphi_{\delta_{23}, n, m}(u ; b)$ as well. In this subsection, we shall consider the $\operatorname{limits}_{\lim _{u \rightarrow \infty}} \phi_{\delta_{123}, n, m}(u ; b)$ and $\lim _{u \rightarrow \infty} \varphi_{\delta_{23}, n, m}(u ; b)$. It is not our objective here to discuss the existence and/or uniqueness of solution to the IDEs given the continuity and limiting conditions in general, but we point out that these are sufficient to yield a unique solution in section 3 when each claim is distributed as a combination of exponentials (see Remark 9). Interested readers are referred to e.g. Mihálykó \& Mihálykó (2011) where conditions for the uniqueness of the solution to an integral equation satisfied by the classical Gerber-Shiu function are analysed. Before providing the limits in Lemmas 2 and 3, we state the following lemma which is a special case of Léveillé \& Garrido (2001, corollary 2.1). 
Lemma 1 Define, for $m \in \mathbb{N}$ and $\delta>0$

$$
\theta_{\delta, m}=E\left[\left(\sum_{k=1}^{\infty} e^{-\delta T_{k}} f\left(Y_{k}\right)\right)^{m}\right]
$$

Then, $\theta_{\delta, m}$ can be computed recursively using, for $m \in \mathbb{N}^{+}$

$$
\theta_{\delta, m}=\frac{\lambda}{m \delta} \sum_{i=0}^{m-1}\left(\begin{array}{c}
m \\
i
\end{array}\right) E\left[f^{m-i}\left(Y_{1}\right)\right] \theta_{\delta, i}
$$

with the starting value $\theta_{\delta, 0}=1$.

Remark 6 From Lemma 1, it is clear that $\theta_{\delta, 1}$ is finite if $E\left[f\left(Y_{1}\right)\right]$ is finite. For $\theta_{\delta, 2}$ to be finite, one requires $E\left[f^{2}\left(Y_{1}\right)\right]$ to be finite (which implies finiteness of $E\left[f\left(Y_{1}\right)\right]$ and hence $\left.\theta_{\delta, 1}\right)$. Recursively, one observes that $\theta_{\delta, m}$ is finite if $E\left[f^{n}\left(Y_{1}\right)\right]$ is finite.

Next, we shall first state $\lim _{u \rightarrow \infty} \varphi_{\delta_{23}, n, m}(u ; b)$ in the following lemma under some mild conditions, and some intermediate results in the proof will be used to identify $\lim _{u \rightarrow \infty} \phi_{\delta_{123, n, m}}(u ; b)$ in the proof of Lemma 2.

Lemma 2 For a given value of $m \in \mathbb{N}$, if $\theta_{\delta_{3}, m}$ defined via (2.11) is finite, then under the positive security loading condition, the limit of $\varphi_{\delta_{23}, n, m}(u ; b)$ is finite and is given by, for $n \in \mathbb{N}$

$$
\lim _{u \rightarrow \infty} \varphi_{\delta_{23}, n, m}(u ; b)=\left(\frac{\alpha}{\delta_{2}}\right)^{n} \theta_{\delta_{3}, m}
$$

Lemma 3 For a given value of $m \in \mathbb{N}$, if the penalty function $w(\cdot, \cdot)$ is bounded and $\theta_{\delta_{3}, m}$ defined via (2.11) is finite, then under the positive security loading condition, the limit of $\phi_{\delta_{123}, n, m}(u ; b)$ is given by, for $n \in \mathbb{N}$

$$
\lim _{u \rightarrow \infty} \phi_{\delta_{123}, n, m}(u ; b)=0
$$

\section{Combination of Exponentials Claims}

In this entire section, we assume that the distribution of each claim amount $Y_{k}$ follows a combination of exponentials with density

$$
p(y)=\sum_{k=1}^{r} q_{k} \mu_{k} e^{-\mu_{k} y}, \quad y>0
$$

where $\sum_{k=1}^{r} q_{k}=1$, and for $k=1,2, \ldots, r$ the parameters $\mu_{k}$ 's are positive and distinct whereas $q_{k}$ 's are non-zero. The class of combinations of exponentials is known to be dense in the set of distributions on $(0, \infty)$, and we refer interested readers to Dufresne (2007) for its fitting. Concerning the quantity $Z_{\delta_{3}}\left(\tau_{b}\right)$ defined via (1.3), we shall focus on its special case $\sum_{k=1}^{N\left(\tau_{b}\right)} e^{-\delta_{3} T_{k}} Y_{k}$ (for $\left.\delta_{3}>0\right)$, which represents the aggregate discounted claims until ruin. Thus, it is assumed that $f(y)=y$ throughout this section. Since all the moments of $Y_{1}$ are finite, the quantity $\theta_{\delta_{3}, m}$ computed via Lemma 1 is also finite for every $m \in \mathbb{N}$ according to Remark 6. In particular, the first two moments of $Y_{1}$ are given by $E\left[Y_{1}\right]=\sum_{k=1}^{r} q_{k} / \mu_{k}$ and $E\left[Y_{1}^{2}\right]=\sum_{k=1}^{r} 2 q_{k} / \mu_{k}^{2}$. Consequently, the random 
variable $\quad \sum_{k=1}^{N\left(\tau_{b}\right)} e^{-\delta_{3} T_{k}} Y_{k} \quad$ is integrable as $\quad \sum_{k=1}^{N\left(\tau_{b}\right)} e^{-\delta_{3} T_{k}} Y_{k} \leq \sum_{k=1}^{\infty} e^{-\delta_{3} T_{k}} Y_{k} \quad$ and $E\left[\sum_{k=1}^{\infty} e^{-\delta_{3} T_{k}} Y_{k}\right]=\theta_{\delta_{3}, 1}$ is finite, and hence $\sum_{k=1}^{N\left(\tau_{b}\right)} e^{-\delta_{3} T_{k}} Y_{k}$ is also integrable on the sets $\left\{\tau_{b}<\infty\right\}$ and $\left\{\tau_{b}=\infty\right\}$. Conditional on ruin occurring, we are interested in correlations involving any two of the aggregate discounted claims until ruin $\sum_{k=1}^{N\left(\tau_{b}\right)} e^{-\delta_{3} T_{k}} Y_{k}$, the discounted dividends until ruin $D_{\delta_{2}}\left(\tau_{b}\right)$ (for $\delta_{2}>0$ ), and the ruin time $\tau_{b}$ (see section 4). Although a penalty function of $w \equiv 1$ is sufficient for our purposes, we shall assume a bounded penalty $w(x, y)=w(y)$ that depends on the deficit $\left|U_{b}\left(\tau_{b}\right)\right|$ but not the surplus before ruin $U_{b}\left(\tau_{b}^{-}\right)$, as this does not complicate our analysis. Note that the limiting condition (2.13) is applicable under the above setting, so is the condition (2.12) as far as the joint moments of the aggregate discounted claims and the discounted dividends are concerned without ruin occurring.

The derivations of explicit expressions for $\phi_{\delta_{123}, n, m}(u ; b)$ and $\varphi_{\delta_{23}, n, m}(u ; b)$ rely on the Lundberg's equation, for $l=1,2$ and $n, m \in \mathbb{N}$

$$
c_{l} s-\left(\lambda+\delta_{1}+n \delta_{2}+m \delta_{3}\right)+\lambda \tilde{p}(s)=0
$$

where $\tilde{p}(s)=\sum_{k=1}^{r} q_{k} \mu_{k} /\left(\mu_{k}+s\right)$ is the Laplace transform of $Y_{1}$. Let $\left\{\rho_{n, m, j}\right\}_{j=1}^{r+1}$ and $\left\{\kappa_{n, m, j}\right\}_{j=1}^{r+1}$ be the $r+1$ roots of (3.2) when $l=1$ and $l=2$, respectively (i.e., the roots $\rho$ 's correspond to the full premium rate $c_{1}=c$, while $\kappa$ 's belong to the net premium rate $\left.c_{2}=c-\alpha\right)$. Each of these two sets of roots are assumed to be distinct (see Remark 7). It is well known that (3.2) has a unique root with non-negative real part (and it is a real root), while the other $r$ roots have negative real parts. When $l=2$, we need to distinguish between these roots, and the non-negative root is denoted by $\kappa_{n, m, r+1}$. (Note that $\kappa_{n, m, r+1}$ is indeed positive except when $\delta_{1}=n=m=0$.) Also, $\rho_{n, m, j}$ and $\kappa_{n, m, j}$ are denoted by $\rho_{n, m, j}^{*}$ and $\kappa_{n, m, j}^{*}$, respectively, when $\delta_{1}=0$. We shall see that the solutions to $\phi_{\delta_{123}, n, m}(u ; b)$ and $\varphi_{\delta_{23}, n, m}(u ; b)$ admit the representations

$$
\begin{gathered}
\phi_{L, \delta_{123}, n, m}(u ; b)=\sum_{i=0}^{m} \sum_{j=1}^{r+1} A_{n, m, i, j} e^{\rho_{n, i, j} u}, \quad 0 \leq u \leq b \\
\phi_{U, \delta_{123}, n, m}(u ; b)=\sum_{i=0}^{n} \sum_{j=0}^{m} \sum_{k=1}^{r} A_{n, m, i, j, k}^{*} e^{\kappa_{i, j, k} u}, \quad u \geq b \\
\varphi_{L, \delta_{23}, n, m}(u ; b)=\sum_{i=0}^{m} \sum_{j=1}^{r+1} C_{n, m, i, j} e^{\rho_{n, i, j}^{*} u}, \quad 0 \leq u \leq b
\end{gathered}
$$

and

$$
\varphi_{U, \delta_{23}, n, m}(u ; b)=\sum_{i=0}^{n} \sum_{j=0}^{m} \sum_{k=1}^{r} C_{n, m, i, j, k}^{*} e^{\kappa_{i, j, k}^{*}}+\left(\frac{\alpha}{\delta_{2}}\right)^{n} \theta_{\delta_{3}, m}, \quad u \geq b
$$

As it has been shown that $\phi_{\delta_{123}, n, m}(u ; b)$ and $\varphi_{\delta_{23}, n, m}(u ; b)$ are continuous for $u \geq 0$, we shall use the domain $0 \leq u \leq b$ and $u \geq b$ for the lower and upper layers, respectively, in the upcoming lemmas and theorems.

Remark 7 In the unlikely case where there are multiple roots to the Lundberg's equation, one or more model parameters (such as $\lambda$ or $\delta_{1}$ ) may be slightly modified such that the roots become distinct. Consequently, one may approximate the ruin quantities of interest by the corresponding ones in a model with distinct roots. For a detailed treatment of multiple Lundberg's roots, we refer interested readers to e.g. Ji \& Zhang (2012). Nevertheless, from e.g. Gerber \& Shiu (2006, equation (A.8)), 
a sufficient condition for the roots to be distinct is that all $q_{k}$ 's in the claim density (3.1) are positive (so that $p(\cdot)$ is a proper mixture of exponentials). Another sufficient condition is that $r=2$. See e.g. equations (7.14) and (7.15) of Gerber et al. (2006) and figures 1 and 2 therein. Under either condition, the roots are real.

3.1. $\phi_{\delta_{123}, n, m}(u ; b)$ and $\varphi_{\delta_{23}, n, m}(u ; b)$ when $n=m=0$

When $n=m=0, \phi_{\delta_{123}, 0,0}(u ; b)=\phi_{\delta_{13}, 0}(u ; b)=\phi_{\delta_{1}}(u ; b)$ is simply the classical Gerber-Shiu function defined in (1.4). Its solution can be obtained from Gerber \& Shiu (2006, appendix B) with minor adjustments (as they considered $w \equiv 1$ ). Since this will be used as a starting point to compute higher moments, the result is stated in the following lemma.

Lemma 4 The classical Gerber-Shiu function $\phi_{\delta_{1}}(u ; b)$ is given by

$$
\phi_{\delta_{1}}(u ; b)=\phi_{L, \delta_{1}}(u ; b)=\sum_{j=1}^{r+1} A_{0,0,0, j} e^{\rho_{0,0, j} u}, \quad 0 \leq u \leq b
$$

and

$$
\phi_{\delta_{1}}(u ; b)=\phi_{U, \delta_{1}}(u ; b)=\sum_{j=1}^{r} A_{0,0,0,0, j}^{*} e^{\kappa_{0,0, j} u}, \quad u \geq b
$$

where $\left\{\rho_{0,0, j}\right\}_{j=1}^{r+1}$ and $\left\{\kappa_{0,0, j}\right\}_{j=1}^{r}$ are Lundberg's roots defined via (3.2). The coefficients $\left\{A_{0,0,0, j}\right\}_{j=1}^{r+1}$ and $\left\{A_{0,0,0,0, j}^{*}\right\}_{j=1}^{r}$ satisfy the $2 r+1$ linear equations which consist of

$$
\begin{gathered}
\sum_{j=1}^{r+1} \frac{A_{0,0,0, j}}{\mu_{k}+\rho_{0,0, j}}=\tilde{w}\left(\mu_{k}\right), \quad k=1,2, \ldots, r \\
\sum_{j=1}^{r+1} \frac{A_{0,0,0, j}}{\mu_{k}+\rho_{0,0, j}} e^{\rho_{0,0, j} b}=\sum_{j=1}^{r} \frac{A_{0,0,0,0, j}^{*}}{\mu_{k}+\kappa_{0,0, j}} e^{\kappa_{0,0, j} b}, \quad k=1,2, \ldots, r
\end{gathered}
$$

and

$$
\sum_{j=1}^{r+1} A_{0,0,0, j} e^{\rho_{0,0, j} b}=\sum_{j=1}^{r} A_{0,0,0,0, j}^{*} e^{\kappa_{0,0, j} b}
$$

where $\tilde{w}(s)=\int_{0}^{\infty} e^{-s y} w(y) d y$ is the Laplace transform of $w(\cdot)$.

When $n=m=0$, it is clear from the definition (1.6) that $\varphi_{\delta_{23}, 0,0}(u ; b)=\varphi(u ; b)$ is the survival probability (i.e., probability that ruin does not occur). Therefore, one has that $\varphi(u ; b)=1-\left.\phi_{\delta_{1}}(u ; b)\right|_{\delta_{1}=0, w \equiv 1}$, where $\left.\phi_{\delta_{1}}(u ; b)\right|_{\delta_{1}=0, w \equiv 1}$ can be computed using Lemma 4 . This leads to the following lemma.

Lemma 5 The survival probability $\varphi(u ; b)$ is given by

$$
\varphi(u ; b)=\varphi_{L}(u ; b)=\sum_{j=1}^{r+1} C_{0,0,0, j} e^{\rho_{0,0, j}^{*}}, \quad 0 \leq u \leq b
$$

and

$$
\varphi(u ; b)=\varphi_{U}(u ; b)=\sum_{j=1}^{r} C_{0,0,0,0, j}^{*} e^{\kappa_{0,0, j}^{*} u}+1, \quad u \geq b
$$


where $\left\{\rho_{0,0, j}^{*}\right\}_{j=1}^{r+1}$ and $\left\{\kappa_{0,0, j}^{*}\right\}_{j=1}^{r}$ are Lundberg's roots defined via (3.2). The coefficients $\left\{C_{0,0,0, j}\right\}_{j=1}^{r+1}$ and $\left\{C_{0,0,0,0, j}^{*}\right\}_{j=1}^{r}$ satisfy the $2 r+1$ linear equations which consist of

$$
\begin{gathered}
\sum_{j=1}^{r+1} \frac{C_{0,0,0, j}}{\mu_{k}+\rho_{0,0, j}^{*}}=0, \quad k=1,2, \ldots, r \\
\sum_{j=1}^{r+1} \frac{C_{0,0,0, j} \mu_{k}}{\mu_{k}+\rho_{0,0, j}^{*}} e^{\rho_{0,0, j}^{*} b}=\sum_{j=1}^{r} \frac{C_{0,0,0,0, j}^{*} \mu_{k}}{\mu_{k}+\kappa_{0,0, j}^{*}} e^{\kappa_{0,0, j}^{*} b}+1, \quad k=1,2, \ldots, r
\end{gathered}
$$

and

$$
\sum_{j=1}^{r+1} C_{0,0,0, j} e^{\rho_{0,0, j}^{*} b}=\sum_{j=1}^{r} C_{0,0,0,0, j}^{*} e^{\kappa_{0,0, j}^{*} b}+1
$$

\section{2. $\phi_{\delta_{123}, n, m}(u ; b)$ and $\varphi_{\delta_{23}, n, m}(u ; b)$ when $n \in \mathbb{N}^{+}$and $m=0$}

We start with the special cases of (1.5) and (1.6) where $n \in \mathbb{N}^{+}$and $m=0$, so that the aggregate claims component $Z_{\delta_{3}}\left(\tau_{b}\right)=\sum_{k=1}^{N\left(\tau_{b}\right)} e^{-\delta_{3} T_{k}} Y_{k}$ is absent in $\phi_{\delta_{123}, n, m}(u ; b)$ and $\varphi_{\delta_{23}, n, m}(u ; b)$. These will be denoted by $\phi_{\delta_{123}, n, 0}(u ; b)=\phi_{\delta_{12, n}}(u ; b)$ and $\varphi_{\delta_{23}, n, 0}(u ; b)=\varphi_{\delta_{2}, n}(u ; b)$, respectively, and given in the following two theorems. The proofs are provided in the Appendix.

Theorem 4 For $n \in \mathbb{N}^{+}$, the Gerber-Shiu function $\phi_{\delta_{12, n}}(u ; b)=\phi_{\delta_{123, n}, 0}(u ; b)$ is given by

$$
\phi_{\delta_{12, n}}(u ; b)=\phi_{L, \delta_{12, n}}(u ; b)=\sum_{j=1}^{r+1} A_{n, 0,0, j} e^{\rho_{n, 0, j} u}, \quad 0 \leq u \leq b
$$

and

$$
\phi_{\delta_{12, n}}(u ; b)=\phi_{U, \delta_{12, n}}(u ; b)=\sum_{i=0}^{n} \sum_{j=1}^{r} A_{n, 0, i, 0, j}^{*} e^{\kappa_{i, 0, j} u}, \quad u \geq b
$$

where $\left\{\rho_{n, 0, j}\right\}_{j=1}^{r+1}$ and $\left\{\kappa_{i, 0, j}\right\}_{j=1}^{r}$ are Lundberg's roots defined via (3.2). The coefficients $\left\{A_{n, 0, i, 0, j}^{*}\right\}_{j=1}^{r}$ (for $i=0,1, \ldots, n-1$ ) can be obtained from

$$
A_{n, 0, i, 0, j}^{*}=\frac{\alpha n}{(n-i) \delta_{2}} A_{n-1,0, i, 0, j}^{*}, \quad i=0,1, \ldots, n-1 ; j=1,2, \ldots, r
$$

while the coefficients $\left\{A_{n, 0,0, j}\right\}_{j=1}^{r+1}$ and $\left\{A_{n, 0, n, 0, j}^{*}\right\}_{j=1}^{r}$ satisfy the $2 r+1$ linear equations which consist of

$$
\begin{gathered}
\sum_{j=1}^{r+1} \frac{A_{n, 0,0, j}}{\mu_{k}+\rho_{n, 0, j}}=0, \quad k=1,2, \ldots, r \\
\sum_{j=1}^{r+1} \frac{A_{n, 0,0, j}}{\mu_{k}+\rho_{n, 0, j}} e^{\rho_{n, 0, j} b}=\sum_{i=0}^{n} \sum_{j=1}^{r} \frac{A_{n, 0, i, 0, j}^{*}}{\mu_{k}+\kappa_{i, 0, j}} e^{\kappa_{i, 0, j} b}, \quad k=1,2, \ldots, r
\end{gathered}
$$

and

$$
\sum_{j=1}^{r+1} A_{n, 0,0, j} e^{\rho_{n, 0, j} b}=\sum_{i=0}^{n} \sum_{j=1}^{r} A_{n, 0, i, 0, j}^{*} e^{\kappa_{i, 0, j} b}
$$

The coefficients $\left\{A_{0,0,0,0, j}^{*}\right\}_{j=1}^{r}$ which form the starting point of the recursion in $n$ can be evaluated using Lemma 4. 
Theorem 5 For $n \in \mathbb{N}^{+}$, the $n$th moment of the discounted dividends without ruin occurring $\varphi_{\delta_{2}, n}(u ; b)=\varphi_{\delta_{23}, n, 0}(u ; b)$ is given by

$$
\varphi_{\delta_{2}, n}(u ; b)=\varphi_{L, \delta_{2}, n}(u ; b)=\sum_{j=1}^{r+1} C_{n, 0,0, j} e^{\rho_{n, 0 j}^{*} u}, \quad 0 \leq u \leq b
$$

and

$$
\varphi_{\delta_{2}, n}(u ; b)=\varphi_{U, \delta_{2}, n}(u ; b)=\sum_{i=0}^{n} \sum_{j=1}^{r} C_{n, 0, i, 0, j}^{*} e^{\kappa_{i, 0, j}^{*} u}+\left(\frac{\alpha}{\delta_{2}}\right)^{n}, \quad u \geq b
$$

where $\left\{\rho_{n, 0, j}^{*}\right\}_{j=1}^{r+1}$ and $\left\{\kappa_{i, 0, j}^{*}\right\}_{j=1}^{r}$ are Lundberg's roots defined via (3.2). The coefficients $\left\{C_{n, 0, i, 0, j}^{*}\right\}_{j=1}^{r}$ (for $i=0,1, \ldots, n-1$ ) can be obtained from

$$
C_{n, 0, i, 0, j}^{*}=\frac{\alpha n}{(n-i) \delta_{2}} C_{n-1,0, i, 0, j}^{*}, \quad i=0,1, \ldots, n-1 ; j=1,2, \ldots, r
$$

while the coefficients $\left\{C_{n, 0,0, j}\right\}_{j=1}^{r+1}$ and $\left\{C_{n, 0, n, 0, j}^{*}\right\}_{j=1}^{r}$ satisfy the $2 r+1$ linear equations which consist of

$$
\begin{gathered}
\sum_{j=1}^{r+1} \frac{C_{n, 0,0, j}}{\mu_{k}+\rho_{n, 0, j}^{*}}=0, \quad k=1,2, \ldots, r \\
\sum_{j=1}^{r+1} \frac{C_{n, 0,0, j}}{\mu_{k}+\rho_{n, 0, j}^{*}} e^{\rho_{n, 0, j}^{*} b}=\sum_{i=0}^{n} \sum_{j=1}^{r} \frac{C_{n, 0, i, 0, j}^{*}}{\mu_{k}+\kappa_{i, 0, j}^{*}} e^{\kappa_{i, 0, j}^{*} b}+\frac{1}{\mu_{k}}\left(\frac{\alpha}{\delta_{2}}\right)^{n}, \quad k=1,2, \ldots, r
\end{gathered}
$$

and

$$
\sum_{j=1}^{r+1} C_{n, 0,0, j} e^{\rho_{n, 0, j}^{*} b}=\sum_{i=0}^{n} \sum_{j=1}^{r} C_{n, 0, i, 0, j}^{*} e^{\kappa_{i, 0, j}^{*} b}+\left(\frac{\alpha}{\delta_{2}}\right)^{n}
$$

The coefficients $\left\{C_{0,0,0,0, j}^{*}\right\}_{j=1}^{r}$, which form the starting point of the recursion in $n$, can be evaluated using Lemma 5.

\section{3. $\phi_{\delta_{123}, n, m}(u ; b)$ and $\varphi_{\delta_{23}, n, m}(u ; b)$ when $n=0$ and $m=1,2$}

We now look at $\phi_{\delta_{123}, n, m}(u ; b)$ when $n=0$, which is denoted by $\phi_{\delta_{123}, 0, m}(u ; b)=\phi_{\delta_{13}, m}(u ; b)$ in section 2.1. The following theorem gives the explicit expression for $\phi_{\delta_{13}, 1}(u ; b)$. The proof is given in the Appendix. It will be seen that the analysis is more involved when $m$ is now non-zero.

Theorem 6 The Gerber-Shiu function $\phi_{\delta_{13}, 1}(u ; b)=\phi_{\delta_{123}, 0,1}(u ; b)$ is given by

$$
\phi_{\delta_{13}, 1}(u ; b)=\phi_{L, \delta_{13}, 1}(u ; b)=\sum_{i=0}^{1} \sum_{j=1}^{r+1} A_{0,1, i, j} e^{\rho_{0, i, j} u}, \quad 0 \leq u \leq b
$$

and

$$
\phi_{\delta_{13}, 1}(u ; b)=\phi_{U, \delta_{13}, 1}(u ; b)=\sum_{i=0}^{1} \sum_{j=1}^{r} A_{0,1,0, i, j}^{*} e^{\kappa_{0, i, j} u}, \quad u \geq b
$$

where $\left\{\rho_{0, i, j}\right\}_{j=1}^{r+1}$ and $\left\{\kappa_{0, i, j}\right\}_{j=1}^{r}$ are Lundberg's roots defined via (3.2). The coefficients $\left\{A_{0,1,0, j}\right\}_{j=1}^{r+1}$ and $\left\{A_{0,1,0,0, j}^{*}\right\}_{j=1}^{r}$ can be computed directly using

$$
A_{0,1,0, j}=\frac{\lambda A_{0,0,0, j}}{\delta_{3}} \sum_{k=1}^{r} \frac{q_{k} \mu_{k}}{\left(\mu_{k}+\rho_{0,0, j}\right)^{2}}, \quad j=1,2, \ldots, r+1
$$


and

$$
A_{0,1,0,0, j}^{*}=\frac{\lambda A_{0,0,0,0, j}^{*}}{\delta_{3}} \sum_{k=1}^{r} \frac{q_{k} \mu_{k}}{\left(\mu_{k}+\kappa_{0,0, j}\right)^{2}}, \quad j=1,2, \ldots, r
$$

where $\left\{A_{0,0,0, j}\right\}_{j=1}^{r+1}$ and $\left\{A_{0,0,0,0, j}^{*}\right\}_{j=1}^{r}$ are obtainable from Lemma 4 . Then the coefficients $\left\{A_{0,1,1, j}\right\}_{j=1}^{r+1}$ and $\left\{A_{0,1,0,1, j}^{*}\right\}_{j=1}^{r}$ can be solved from the $2 r+1$ linear equations which consist of

$$
\begin{gathered}
\sum_{i=0}^{1} \sum_{j=1}^{r+1} \frac{A_{0,1, i, j}}{\mu_{k}+\rho_{0, i, j}}+\sum_{j=1}^{r+1} \frac{A_{0,0,0, j}}{\left(\mu_{k}+\rho_{0,0, j}\right)^{2}}=\mathcal{T}_{\mu_{k}}^{2} w(0), \quad k=1,2, \ldots, r \\
\sum_{i=0}^{1} \sum_{j=1}^{r+1} \frac{A_{0,1, i, j}}{\mu_{k}+\rho_{0, i, j}} e^{\rho_{0, i, j} b}+\sum_{j=1}^{r+1} \frac{A_{0,0,0, j}}{\left(\mu_{k}+\rho_{0,0, j}\right)^{2}} e^{\rho_{0,0, j} b}=\sum_{i=0}^{1} \sum_{j=1}^{r} \frac{A_{0,1,0, i, j}^{*}}{\mu_{k}+\kappa_{0, i, j}} e^{\kappa_{0, i, j} b}+\sum_{j=1}^{r} \frac{A_{0,0,0,0, j}^{*}}{\left(\mu_{k}+\kappa_{0,0, j}\right)^{2}} e^{\kappa_{0,0, j} b} \\
k=1,2, \ldots, r
\end{gathered}
$$

and

$$
\sum_{i=0}^{1} \sum_{j=1}^{r+1} A_{0,1, i, j} e^{\rho_{0, i, j} b}=\sum_{i=0}^{1} \sum_{j=1}^{r} A_{0,1,0, i, j}^{*} e^{\kappa_{0, i, j} b}
$$

where $\mathcal{T}_{\mu_{k}}^{2} w(0)=\int_{0}^{\infty} y e^{-\mu_{k} y} w(y) d y$ in (3.29) is the notation of a double Dickson-Hipp operator (see Dickson \& Hipp, 2001; Li \& Garrido, 2004).

The next theorem gives the result for $\phi_{\delta_{13}, 2}(u ; b)$. Since the logic of the derivation is identical to that of Theorem 6 (although it is more tedious), the proof is omitted. Note that (3.32)-(3.35) concerning the Gerber-Shiu function $\phi_{L, \delta_{13}, 2}(u ; b)$ in the lower layer are direct consequences of (39)-(42) in theorem 7 of Cheung et al. (2015) (see Remark 4).

Theorem 7 The Gerber-Shiu function $\phi_{\delta_{13}, 2}(u ; b)=\phi_{\delta_{123}, 0,2}(u ; b)$ is given by

and

$$
\phi_{\delta_{13}, 2}(u ; b)=\phi_{L, \delta_{13}, 2}(u ; b)=\sum_{i=0}^{2} \sum_{j=1}^{r+1} A_{0,2, i, j} e^{\rho_{0, i, j} u}, \quad 0 \leq u \leq b
$$

$$
\phi_{\delta_{13}, 2}(u ; b)=\phi_{U, \delta_{13}, 2}(u ; b)=\sum_{i=0}^{2} \sum_{j=1}^{r} A_{0,2,0, i, j}^{*} e^{\kappa_{0, i, j} u}, \quad u \geq b
$$

where $\left\{\rho_{0, i, j}\right\}_{j=1}^{r+1}$ and $\left\{\kappa_{0, i, j}\right\}_{j=1}^{r}$ are Lundberg's roots defined via (3.2). For $i=0$, 1 , the coefficients $\left\{A_{0,2, i, j}\right\}_{j=1}^{r+1}$ and $\left\{A_{0,2,0, i, j}^{*}\right\}_{j=1}^{r}$ can be computed directly using

$$
\begin{gathered}
A_{0,2,0, j}=\frac{\lambda A_{0,1,0, j}}{\delta_{3}} \sum_{k=1}^{r} \frac{q_{k} \mu_{k}}{\left(\mu_{k}+\rho_{0,0, j}\right)^{2}}+\frac{\lambda A_{0,0,0, j}}{\delta_{3}} \sum_{k=1}^{r} \frac{q_{k} \mu_{k}}{\left(\mu_{k}+\rho_{0,0, j}\right)^{3}}, \quad j=1,2, \ldots, r+1 \\
A_{0,2,1, j}=\frac{2 \lambda A_{0,1,1, j}}{\delta_{3}} \sum_{k=1}^{r} \frac{q_{k} \mu_{k}}{\left(\mu_{k}+\rho_{0,1, j}\right)^{2}}, \quad j=1,2, \ldots, r+1 \\
A_{0,2,0,0, j}^{*}=\frac{\lambda A_{0,1,0,0, j}^{*}}{\delta_{3}} \sum_{k=1}^{r} \frac{q_{k} \mu_{k}}{\left(\mu_{k}+\kappa_{0,0, j}\right)^{2}}+\frac{\lambda A_{0,0,0,0, j}^{*}}{\delta_{3}} \sum_{k=1}^{r} \frac{q_{k} \mu_{k}}{\left(\mu_{k}+\kappa_{0,0, j}\right)^{3}}, \quad j=1,2, \ldots, r
\end{gathered}
$$


and

$$
A_{0,2,0,1, j}^{*}=\frac{2 \lambda A_{0,1,0,1, j}^{*}}{\delta_{3}} \sum_{k=1}^{r} \frac{q_{k} \mu_{k}}{\left(\mu_{k}+\kappa_{0,1, j}\right)^{2}}, \quad j=1,2, \ldots, r
$$

where $\left\{A_{0,0,0, j}\right\}_{j=1}^{r+1}$ and $\left\{A_{0,0,0,0, j}^{*}\right\}_{j=1}^{r}$ are obtainable from Lemma 4, while for $i=0$, 1 , the coefficients $\left\{A_{0,1, i, j}\right\}_{j=1}^{r+1}$ and $\left\{A_{0,1,0, i, j}^{*}\right\}_{j=1}^{r}$ are obtainable from Theorem 6. Then, the coefficients $\left\{A_{0,2,2, j}\right\}_{j=1}^{r+1}$ and $\left\{A_{0,2,0,2, j}^{*}\right\}_{j=1}^{r}$ can be solved from the $2 r+1$ linear equations which consist of

$$
\begin{aligned}
& \sum_{i=0}^{2} \sum_{j=1}^{r+1} \frac{A_{0,2, i, j}}{\mu_{k}+\rho_{0, i, j}}+\sum_{i=0}^{1} \sum_{j=1}^{r+1} \frac{2 A_{0,1, i, j}}{\left(\mu_{k}+\rho_{0, i, j}\right)^{2}}+\sum_{j=1}^{r+1} \frac{2 A_{0,0,0, j}}{\left(\mu_{k}+\rho_{0,0, j}\right)^{3}}=2 \mathcal{T}_{\mu_{k}}^{3} w(0), \quad k=1,2, \ldots, r \\
& \sum_{i=0}^{2} \sum_{j=1}^{r+1} \frac{A_{0,2, i, j}}{\mu_{k}+\rho_{0, i, j}} e^{\rho_{0, i j} b}+\sum_{i=0}^{1} \sum_{j=1}^{r+1} \frac{2 A_{0,1, i, j}}{\left(\mu_{k}+\rho_{0, i, j}\right)^{2}} e^{\rho_{0, i, j} b}+\sum_{j=1}^{r+1} \frac{2 A_{0,0,0, j}}{\left(\mu_{k}+\rho_{0,0, j}\right)^{3}} e^{\rho_{0,0, j} b} \\
& \quad=\sum_{i=0}^{2} \sum_{j=1}^{r} \frac{A_{0,2,0, i, j}^{*}}{\mu_{k}+\kappa_{0, i, j}} e^{\kappa_{0, i, j} b}+\sum_{i=0}^{1} \sum_{j=1}^{r} \frac{2 A_{0,1,0, i, j}^{*}}{\left(\mu_{k}+\kappa_{0, i, j}\right)^{2}} e^{\kappa_{0, i, j} b}+\sum_{j=1}^{r} \frac{2 A_{0,0,0,0, j}^{*}}{\left(\mu_{k}+\kappa_{0,0, j}\right)^{3}} e^{\kappa_{0,0, j} b}, \quad k=1,2, \ldots, r
\end{aligned}
$$

and

$$
\sum_{i=0}^{2} \sum_{j=1}^{r+1} A_{0,2, i, j} e^{\rho_{0, i, j} b}=\sum_{i=0}^{2} \sum_{j=1}^{r} A_{0,2,0, i, j}^{*} e^{\kappa_{0, i, j} b}
$$

where $\mathcal{T}_{\mu_{k}}^{3} w(0)=\int_{0}^{\infty}\left(y^{2} e^{-\mu_{k} y} / 2\right) w(y) d y$ in (3.35) is the notation of a triple Dickson-Hipp operator.

Concerning the first two moments of the aggregate discounted claims without ruin occurring (denoted by $\varphi_{\delta_{23}, 0, m}(u ; b)=\varphi_{\delta_{3}, m}(u ; b)$ for $m=1,2$ ), the results are stated in the following theorems. Their proofs follow closely those of Theorems 6 and 7 and are omitted.

Theorem 8 The expected aggregate discounted claims $\varphi_{\delta_{3}, 1}(u ; b)=\varphi_{\delta_{23}, 0,1}(u ; b)$ without ruin occurring is given by

$$
\varphi_{\delta_{3}, 1}(u ; b)=\varphi_{L, \delta_{3}, 1}(u ; b)=\sum_{i=0}^{1} \sum_{j=1}^{r+1} C_{0,1, i, j} e^{\rho_{0, i, j}^{*} u}, \quad 0 \leq u \leq b,
$$

and

$$
\varphi_{\delta_{3}, 1}(u ; b)=\varphi_{U, \delta_{3}, 1}(u ; b)=\sum_{i=0}^{1} \sum_{j=1}^{r} C_{0,1,0, i, j}^{*} e^{\kappa_{0, i, j}^{*} u}+\frac{\lambda E\left[Y_{1}\right]}{\delta_{3}}, \quad u \geq b
$$

where $\left\{\rho_{0, i, j}^{*}\right\}_{j=1}^{r+1}$ and $\left\{\kappa_{0, i, j}^{*}\right\}_{j=1}^{r}$ are Lundberg's roots defined via (3.2). The coefficients $\left\{C_{0,1,0, j}\right\}_{j=1}^{r+1}$ and $\left\{C_{0,1,0,0, j}^{*}\right\}_{j=1}^{r}$ can be computed directly using

$$
C_{0,1,0, j}=\frac{\lambda C_{0,0,0, j}}{\delta_{3}} \sum_{k=1}^{r} \frac{q_{k} \mu_{k}}{\left(\mu_{k}+\rho_{0,0, j}^{*}\right)^{2}}, \quad j=1,2, \ldots, r+1
$$

and

$$
C_{0,1,0,0, j}^{*}=\frac{\lambda C_{0,0,0,0, j}^{*}}{\delta_{3}} \sum_{k=1}^{r} \frac{q_{k} \mu_{k}}{\left(\mu_{k}+\kappa_{0,0, j}^{*}\right)^{2}}, \quad j=1,2, \ldots, r
$$


where $\left\{C_{0,0,0, j}\right\}_{j=1}^{r+1}$ and $\left\{C_{0,0,0,0, j}^{*}\right\}_{j=1}^{r}$ are obtainable from Lemma 5. Then the coefficients $\left\{C_{0,1,1, j}\right\}_{j=1}^{r+1}$ and $\left\{C_{0,1,0,1, j}^{*}\right\}_{j=1}^{r}$ can be solved from the $2 r+1$ linear equations which consist of

$$
\begin{gathered}
\sum_{i=0}^{1} \sum_{j=1}^{r+1} \frac{C_{0,1, i, j}}{\mu_{k}+\rho_{0, i, j}^{*}}+\sum_{j=1}^{r+1} \frac{C_{0,0,0, j}}{\left(\mu_{k}+\rho_{0,0, j}^{*}\right)^{2}}=0, \quad k=1,2, \ldots, r \\
\sum_{i=0}^{1} \sum_{j=1}^{r+1} \frac{C_{0,1, i, j}}{\mu_{k}+\rho_{0, i, j}^{*}} e^{\rho_{0, i, j}^{*} b}+\sum_{j=1}^{r+1} \frac{C_{0,0,0, j}}{\left(\mu_{k}+\rho_{0,0, j}^{*}\right)^{2}} e^{\rho_{0,0, j}^{*} b} \\
=\sum_{i=0}^{1} \sum_{j=1}^{r} \frac{C_{0,1,0, i, j}^{*}}{\mu_{k}+\kappa_{0, i, j}^{*}} e^{\kappa_{0, i, j}^{*} b}+\sum_{j=1}^{r} \frac{C_{0,0,0,0, j}^{*}}{\left(\mu_{k}+\kappa_{0,0, j}^{*}\right)^{2}} e^{\kappa_{0,0, j}^{*} b}+\frac{\lambda E\left[Y_{1}\right]}{\mu_{k} \delta_{3}}+\frac{1}{\mu_{k}^{2}}, \quad k=1,2, \ldots, r
\end{gathered}
$$

and

$$
\sum_{i=0}^{1} \sum_{j=1}^{r+1} C_{0,1, i, j} e^{\rho_{0, i, j}^{*} b}=\sum_{i=0}^{1} \sum_{j=1}^{r} C_{0,1,0, i, j}^{*} e^{\kappa_{0, i, j}^{*} b}+\frac{\lambda E\left[Y_{1}\right]}{\delta_{3}}
$$

Theorem 9 The second moment of the aggregate discounted claims $\varphi_{\delta_{3}, 2}(u ; b)=\varphi_{\delta_{23}, 0,2}(u ; b)$ without ruin occurring is given by

and

$$
\varphi_{\delta_{3}, 2}(u ; b)=\varphi_{L, \delta_{3}, 2}(u ; b)=\sum_{i=0}^{2} \sum_{j=1}^{r+1} C_{0,2, i, j} e^{\rho_{0, i, j}^{*} u}, \quad 0 \leq u \leq b
$$

$$
\varphi_{\delta_{3}, 2}(u ; b)=\varphi_{U, \delta_{3}, 2}(u ; b)=\sum_{i=0}^{2} \sum_{j=1}^{r} C_{0,2,0, i, j}^{*} e^{\kappa_{0, i j}^{*} u}+\theta_{\delta_{3}, 2}, \quad u \geq b
$$

where $\left\{\rho_{0, i, j}^{*}\right\}_{j=1}^{r+1}$ and $\left\{\kappa_{0, i, j}^{*}\right\}_{j=1}^{r}$ are Lundberg's roots defined via (3.2). The constant term $\theta_{\delta_{3}, 2}$ in (3.36) can be evaluated by Lemma 1 as $\theta_{\delta_{3}, 2}=\lambda\left(2 E\left[Y_{1}\right] \theta_{\delta_{3}, 1}+E\left[Y_{1}^{2}\right]\right) /\left(2 \delta_{3}\right)$ with $\theta_{\delta_{3}, 1}=\lambda E\left[Y_{1}\right] / \delta_{3}$. For $i=0,1$, the coefficients $\left\{C_{0,2, i, j}\right\}_{j=1}^{r+1}$ and $\left\{C_{0,2,0, i, j}^{*}\right\}_{j=1}^{r}$ can be computed directly using

$$
\begin{gathered}
C_{0,2,0, j}=\frac{\lambda C_{0,1,0, j}}{\delta_{3}} \sum_{k=1}^{r} \frac{q_{k} \mu_{k}}{\left(\mu_{k}+\rho_{0,0, j}^{*}\right)^{2}}+\frac{\lambda C_{0,0,0, j}}{\delta_{3}} \sum_{k=1}^{r} \frac{q_{k} \mu_{k}}{\left(\mu_{k}+\rho_{0,0, j}^{*}\right)^{3}}, \quad j=1,2, \ldots, r+1 \\
C_{0,2,1, j}=\frac{2 \lambda C_{0,1,1, j}}{\delta_{3}} \sum_{k=1}^{r} \frac{q_{k} \mu_{k}}{\left(\mu_{k}+\rho_{0,1, j}^{*}\right)^{2}}, \quad j=1,2, \ldots, r+1 \\
C_{0,2,0,0, j}^{*}=\frac{\lambda C_{0,1,0,0, j}^{*}}{\delta_{3}} \sum_{k=1}^{r} \frac{q_{k} \mu_{k}}{\left(\mu_{k}+\kappa_{0,0, j}^{*}\right)^{2}}+\frac{\lambda C_{0,0,0,0, j}^{*}}{\delta_{3}} \sum_{k=1}^{r} \frac{q_{k} \mu_{k}}{\left(\mu_{k}+\kappa_{0,0, j}^{*}\right)^{3}}, \quad j=1,2, \ldots, r
\end{gathered}
$$

and

$$
C_{0,2,0,1, j}^{*}=\frac{2 \lambda C_{0,1,0,1, j}^{*}}{\delta_{3}} \sum_{k=1}^{r} \frac{q_{k} \mu_{k}}{\left(\mu_{k}+\kappa_{0,1, j}^{*}\right)^{2}}, \quad j=1,2, \ldots, r
$$

where $\left\{C_{0,0,0, j}\right\}_{j=1}^{r+1}$ and $\left\{C_{0,0,0,0, j}^{*}\right\}_{j=1}^{r}$ are obtainable from Lemma 5, while for $i=0,1$ the coefficients $\left\{C_{0,1, i, j}\right\}_{j=1}^{r+1}$ and $\left\{C_{0,1,0, i, j}^{*}\right\}_{j=1}^{r}$ are obtainable from Theorem 8. Then, the coefficients $\left\{C_{0,2,2, j}\right\}_{j=1}^{r+1}$ and $\left\{C_{0,2,0,2, j}^{*}\right\}_{j=1}^{r}$ can be solved from the $2 r+1$ linear equations which consist of

$$
\sum_{i=0}^{2} \sum_{j=1}^{r+1} \frac{C_{0,2, i, j}}{\mu_{k}+\rho_{0, i, j}^{*}}+\sum_{i=0}^{1} \sum_{j=1}^{r+1} \frac{2 C_{0,1, i, j}}{\left(\mu_{k}+\rho_{0, i, j}^{*}\right)^{2}}+\sum_{j=1}^{r+1} \frac{2 C_{0,0,0, j}}{\left(\mu_{k}+\rho_{0,0, j}^{*}\right)^{3}}=0, \quad k=1,2, \ldots, r
$$




$$
\begin{gathered}
\sum_{i=0}^{2} \sum_{j=1}^{r+1} \frac{C_{0,2, i, j}}{\mu_{k}+\rho_{0, i, j}^{*}} e^{\rho_{0, i, j}^{*} b}+\sum_{i=0}^{1} \sum_{j=1}^{r+1} \frac{2 C_{0,1, i, j}}{\left(\mu_{k}+\rho_{0, i, j}^{*}\right)^{2}} e^{\rho_{0, i, j}^{*} b}+\sum_{j=1}^{r+1} \frac{2 C_{0,0,0, j}}{\left(\mu_{k}+\rho_{0,0, j}^{*}\right)^{3}} e^{\rho_{0,0, j}^{*} b}-\frac{\theta_{\delta_{3}, 2}}{\mu_{k}}-\frac{2 \theta_{\delta_{3}, 1}}{\mu_{k}^{2}}-\frac{2}{\mu_{k}^{3}} \\
=\sum_{i=0}^{2} \sum_{j=1}^{r} \frac{C_{0,2,0, i, j}^{*}}{\mu_{k}+\kappa_{0, i, j}^{*}} e^{\kappa_{0, i, j}^{*} b}+\sum_{i=0}^{1} \sum_{j=1}^{r} \frac{2 C_{0,1,0, i, j}^{*}}{\left(\mu_{k}+\kappa_{0, i, j}^{*}\right)^{2}} e^{\kappa_{0, i, j}^{*} b}+\sum_{j=1}^{r} \frac{2 C_{0,0,0,0, j}^{*}}{\left(\mu_{k}+\kappa_{0,0, j}^{*}\right)^{3}} e^{\kappa_{0,0, j}^{*} b}, \quad k=1,2, \ldots, r
\end{gathered}
$$

and

$$
\sum_{i=0}^{2} \sum_{j=1}^{r+1} C_{0,2, i, j} e^{\rho_{0, i j}^{*} b}=\sum_{i=0}^{2} \sum_{j=1}^{r} C_{0,2,0, i, j}^{*} e^{\kappa_{0, i, j}^{*} b}+\theta_{\delta_{3}, 2}
$$

3.4. $\phi_{\delta_{123}, n, m}(u ; b)$ and $\varphi_{\delta_{23}, n, m}(u ; b)$ when $n=m=1$

In the next two theorems, the procedures to find $\phi_{\delta_{123,1,1}}(u ; b)$ and $\varphi_{\delta_{23,1,1}}(u ; b)$ are provided. These two quantities will be useful for computing the covariance (and hence correlation) between the discounted dividends $D_{\delta_{2}}\left(\tau_{b}\right)$ and the aggregate discounted claims $Z_{\delta_{3}}\left(\tau_{b}\right)=\sum_{k=1}^{N\left(\tau_{b}\right)} e^{-\delta_{3} T_{k}} Y_{k}$. Again, (3.37), (3.39), and (3.43) follow directly from Cheung et al. (2015, equations (50)-(52)).

Theorem 10 The Gerber-Shiu function $\phi_{\delta_{123}, 1,1}(u ; b)$ is given by

$$
\phi_{\delta_{123}, 1,1}(u ; b)=\phi_{L, \delta_{123}, 1,1}(u ; b)=\sum_{i=0}^{1} \sum_{j=1}^{r+1} A_{1,1, i, j} e^{\rho_{1, i j} u}, \quad 0 \leq u \leq b
$$

and

$$
\phi_{\delta_{123}, 1,1}(u ; b)=\phi_{U, \delta_{123}, 1,1}(u ; b)=\sum_{l=0}^{1} \sum_{i=0}^{1} \sum_{j=1}^{r} A_{1,1, l, i, j}^{*} e^{\kappa_{l, i, j} u}, \quad u \geq b
$$

where $\left\{\rho_{1, i, j}\right\}_{j=1}^{r+1}$ and $\left\{\kappa_{l, i, j}\right\}_{j=1}^{r}$ are Lundberg's roots defined via (3.2). The coefficients $\left\{A_{1,1,0, j}\right\}_{j=1}^{r+1}$, $\left\{A_{1,1,0, i, j}^{*}\right\}_{j=1}^{r}$ (for $\left.i=0,1\right)$ and $\left\{A_{1,1,1,0, j}^{*}\right\}_{j=1}^{r}$ can be computed directly using

$$
\begin{gathered}
A_{1,1,0, j}=\frac{\lambda A_{1,0,0, j}}{\delta_{3}} \sum_{k=1}^{r} \frac{q_{k} \mu_{k}}{\left(\mu_{k}+\rho_{1,0, j}\right)^{2}}, \quad j=1,2, \ldots, r+1 \\
A_{1,1,0,0, j}^{*}=\frac{\alpha A_{0,1,0,0, j}^{*}}{\delta_{2}+\delta_{3}}+\frac{\lambda A_{1,0,0,0, j}^{*}}{\delta_{2}+\delta_{3}} \sum_{k=1}^{r} \frac{q_{k} \mu_{k}}{\left(\mu_{k}+\kappa_{0,0, j}\right)^{2}}, \quad j=1,2, \ldots, r \\
A_{1,1,0,1, j}^{*}=\frac{\alpha}{\delta_{2}} A_{0,1,0,1, j}^{*}, \quad j=1,2, \ldots, r
\end{gathered}
$$

and

$$
A_{1,1,1,0, j}^{*}=\frac{\lambda A_{1,0,1,0, j}^{*}}{\delta_{3}} \sum_{k=1}^{r} \frac{q_{k} \mu_{k}}{\left(\mu_{k}+\kappa_{1,0, j}\right)^{2}}, \quad j=1,2, \ldots, r
$$

where $\left\{A_{1,0,0, j}\right\}_{j=1}^{r+1}$ and $\left\{A_{1,0, i, 0, j}^{*}\right\}_{j=1}^{r}$ (for $i=0,1$ ) are obtainable from Theorem 4, while $\left\{A_{0,1,0, i, j}^{*}\right\}_{j=1}^{r}$ (for $i=0,1$ ) are obtainable from Theorem 6. Then the coefficients $\left\{A_{1,1,1, j}\right\}_{j=1}^{r+1}$ and $\left\{A_{1,1,1,1, j}^{*}\right\}_{j=1}^{r}$ can be solved from the $2 r+1$ linear equations which consist of

$$
\sum_{i=0}^{1} \sum_{j=1}^{r+1} \frac{A_{1,1, i, j}}{\mu_{k}+\rho_{1, i, j}}+\sum_{j=1}^{r+1} \frac{A_{1,0,0, j}}{\left(\mu_{k}+\rho_{1,0, j}\right)^{2}}=0, \quad k=1,2, \ldots, r
$$




$$
\begin{aligned}
& \sum_{i=0}^{1} \sum_{j=1}^{r+1} \frac{A_{1,1, i, j}}{\mu_{k}+\rho_{1, i, j}} e^{\rho_{1, i, j} b}+\sum_{j=1}^{r+1} \frac{A_{1,0,0, j}}{\left(\mu_{k}+\rho_{1,0, j}\right)^{2}} e^{\rho_{1,0, j} b} \\
& =\sum_{l=0}^{1} \sum_{i=0}^{1} \sum_{j=1}^{r} \frac{A_{1,1, l, i, j}^{*}}{\mu_{k}+\kappa_{l, i, j}} e^{\kappa_{l, i, j} b}+\sum_{i=0}^{1} \sum_{j=1}^{r} \frac{A_{1,0, i, 0, j}^{*}}{\left(\mu_{k}+\kappa_{i, 0, j}\right)^{2}} e^{\kappa_{i, 0, j} b}, \quad k=1,2, \ldots, r
\end{aligned}
$$

and

$$
\sum_{i=0}^{1} \sum_{j=1}^{r+1} A_{1,1, i, j} e^{\rho_{1, i, j} b}=\sum_{l=0}^{1} \sum_{i=0}^{1} \sum_{j=1}^{r} A_{1,1, l, i, j}^{*} e^{\kappa_{l, i, j} b}
$$

Theorem 11 The first joint moment of the discounted dividends and the aggregate discounted claims $\varphi_{\delta_{23}, 1,1}(u ; b)$ without ruin occurring is given by

$$
\varphi_{\delta_{23}, 1,1}(u ; b)=\varphi_{L, \delta_{23}, 1,1}(u ; b)=\sum_{i=0}^{1} \sum_{j=1}^{r+1} C_{1,1, i, j} e^{\rho_{1, i j}^{*} u}, \quad 0 \leq u \leq b
$$

and

$$
\varphi_{\delta_{23}, 1,1}(u ; b)=\varphi_{U, \delta_{23}, 1,1}(u ; b)=\sum_{l=0}^{1} \sum_{i=0}^{1} \sum_{j=1}^{r} C_{1,1, l, i, j}^{*} e^{\kappa_{l, i, j}^{*} u}+\frac{\alpha \lambda E\left[Y_{1}\right]}{\delta_{2} \delta_{3}}, \quad u \geq b
$$

where $\left\{\rho_{1, i, j}^{*}\right\}_{j=1}^{r+1}$ and $\left\{\kappa_{l, i, j}^{*}\right\}_{j=1}^{r}$ are Lundberg's roots defined via (3.2). The coefficients $\left\{C_{1,1,0, j}\right\}_{j=1}^{r+1}$, $\left\{C_{1,1,0, i, j}^{*}\right\}_{j=1}^{r}$ (for $\left.i=0,1\right)$ and $\left\{C_{1,1,1,0, j}^{*}\right\}_{j=1}^{r}$ can be computed directly using

$$
\begin{aligned}
& C_{1,1,0, j}=\frac{\lambda C_{1,0,0, j}}{\delta_{3}} \sum_{k=1}^{r} \frac{q_{k} \mu_{k}}{\left(\mu_{k}+\rho_{1,0, j}^{*}\right)^{2}}, j=1,2, \ldots, r+1 \\
& C_{1,1,0,0, j}^{*}=\frac{\alpha C_{0,1,0,0, j}^{*}}{\delta_{2}+\delta_{3}}+\frac{\lambda C_{1,0,0,0, j}^{*}}{\delta_{2}+\delta_{3}} \sum_{k=1}^{r} \frac{q_{k} \mu_{k}}{\left(\mu_{k}+\kappa_{0,0, j}^{*}\right)^{2}}, j=1,2, \ldots, r \\
& C_{1,1,0,1, j}^{*}=\frac{\alpha}{\delta_{2}} C_{0,1,0,1, j}^{*}, \quad j=1,2, \ldots, r
\end{aligned}
$$

and

$$
C_{1,1,1,0, j}^{*}=\frac{\lambda C_{1,0,1,0, j}^{*}}{\delta_{3}} \sum_{k=1}^{r} \frac{q_{k} \mu_{k}}{\left(\mu_{k}+\kappa_{1,0, j}^{*}\right)^{2}}, \quad j=1,2, \ldots, r
$$

where $\left\{C_{1,0,0, j}\right\}_{j=1}^{r+1}$ and $\left\{C_{1,0, i, 0, j}^{*}\right\}_{j=1}^{r}$ (for $\left.i=0,1\right)$ are obtainable from Theorem 5, while $\left\{C_{0,1,0, i, j}^{*}\right\}_{j=1}^{r}$ (for $i=0,1$ ) are obtainable from Theorem 8 . Then the coefficients $\left\{C_{1,1,1, j}\right\}_{j=1}^{r+1}$ and $\left\{C_{1,1,1,1, j}^{*}\right\}_{j=1}^{r}$ can be solved from the $2 r+1$ linear equations which consist of

$$
\begin{aligned}
& \sum_{i=0}^{1} \sum_{j=1}^{r+1} \frac{C_{1,1, i, j}}{\mu_{k}+\rho_{1, i, j}^{*}}+\sum_{j=1}^{r+1} \frac{C_{1,0,0, j}}{\left(\mu_{k}+\rho_{1,0, j}^{*}\right)^{2}}=0, \quad k=1,2, \ldots, r \\
& \sum_{i=0}^{1} \sum_{j=1}^{r+1} \frac{C_{1,1, i, j}}{\mu_{k}+\rho_{1, i, j}^{*}} e^{\rho_{1, i, j}^{*} b}+\sum_{j=1}^{r+1} \frac{C_{1,0,0, j}}{\left(\mu_{k}+\rho_{1,0, j}^{*}\right)^{2}} e^{\rho_{1,0, j}^{*} b} \\
& =\sum_{l=0}^{1} \sum_{i=0}^{1} \sum_{j=1}^{r} \frac{C_{1,1, l, i, j}^{*}}{\mu_{k}+\kappa_{l, i, j}^{*}} e^{\kappa_{l, i, j}^{*} b}+\sum_{i=0}^{1} \sum_{j=1}^{r} \frac{C_{1,0, i, 0, j}^{*}}{\left(\mu_{k}+\kappa_{i, 0, j}^{*}\right)^{2}} e^{\kappa_{i, 0, j}^{*} b}+\frac{\alpha \lambda E\left[Y_{1}\right]}{\mu_{k} \delta_{2} \delta_{3}}+\frac{\alpha}{\mu_{k}^{2} \delta_{2}}, \quad k=1,2, \ldots, r
\end{aligned}
$$


and

$$
\sum_{i=0}^{1} \sum_{j=1}^{r+1} C_{1,1, i, j} e^{\rho_{1, i, j}^{*} b}=\sum_{l=0}^{1} \sum_{i=0}^{1} \sum_{j=1}^{r} C_{1,1, l, i, j}^{*} e^{\kappa_{l, i, j}^{*} b}+\frac{\alpha \lambda E\left[Y_{1}\right]}{\delta_{2} \delta_{3}}
$$

Remark 8 From the statements of the theorems in this section, it is important to note the recursive nature of the determination of the coefficients involved in the solution forms (3.3)-(3.6). For example, the computational steps required to calculate $\phi_{\delta_{123}, 1,1}(u ; b)$ via Theorem 10 involve the use of earlier lemma and theorems, and these are summarised as follows:

1. For each fixed $l=1,2$ and $n, m=0,1$, we solve the Lundberg's equation (3.2), which has $r+1$ roots, i.e. the equation is solved eight times. When $l=1$ the roots are denoted by $\left\{\rho_{n, m, j}\right\}_{j=1}^{r+1}$, but when $l=2$ they are denoted by $\left\{\kappa_{n, m, j}\right\}_{j=1}^{r+1}$ and the non-negative root $\kappa_{n, m, r+1}$ is discarded.

2. Obtain $\left\{A_{0,0,0, j}\right\}_{j=1}^{r+1}$ and $\left\{A_{0,0,0,0, j}^{*}\right\}_{j=1}^{r}$ from Lemma 4 by solving the linear equations (3.9)-(3.11).

3. Apply the special case of Theorem 4 under $n=1$, where $\left\{A_{1,0,0, j}\right\}_{j=1}^{r+1}$ and $\left\{A_{1,0, i, 0, j}^{*}\right\}_{j=1}^{r}$ (for $i=0$, 1) are computed from (3.15) and the linear system (3.16)-(3.18).

4. Use Theorem 6 to compute $\left\{A_{0,1, i, j}\right\}_{j=1}^{r+1}$ and $\left\{A_{0,1,0, i, j}^{*}\right\}_{j=1}^{r}$ (both for $\left.i=0,1\right)$ via (3.27) and (3.28) along with the linear system (3.29)-(3.31). (Although $A_{0,1, i, j}$ 's are not needed in the next step, they have to be determined together with $A_{0,1,0,1, j}^{*}$ 's.)

5. Utilise Theorem 10 to calculate $\left\{A_{1,1, i, j}\right\}_{j=1}^{r+1}$ (for $\left.i=0,1\right)$ and $\left\{A_{1,1, l, i, j}^{*}\right\}_{j=1}^{r}$ (for $l, i=0,1$ ) via (3.39)-(3.42) and the linear system (3.43)-(3.45), so that $\phi_{\delta_{123}, 1,1}(u ; b)$ is finally evaluated with (3.37) and (3.38).

Remark 9 Following the proof of Theorem 4 in the Appendix, it is noted that the exact value of the limit $\lim _{u \rightarrow \infty} \phi_{\delta_{12}, 1}(u ; b)$ is indeed not required for deriving the full solution to $\phi_{\delta_{12}, 1}(u ; b)$. We only need the finiteness of the limit to conclude that the coefficient of the exponential term $e^{\kappa_{1,0, r+1} u}$ is 0 as $\kappa_{1,0, r+1}>0$, and then from (3.14) the $\operatorname{limit}_{\lim _{u \rightarrow \infty}} \phi_{\delta_{12}, 1}(u ; b)$ must equal 0 as $\kappa_{1,0, j}$ has negative real part for $j=1,2, \ldots, r$. This is also true for the proof of Theorem 5, except that the final term in (3.20) (when $n=1$ ) obtainable via equating the constant term in (A.17) is non-zero (and it automatically satisfies the limiting value $\lim _{u \rightarrow \infty} \varphi_{\delta_{2}, 1}(u ; b)$ suggested by Lemma 2$)$. The same comments are also applicable to Theorems 6-11 (although the proofs of Theorems 7-11 have been omitted).

\section{Numerical Examples}

In this section, the results in section 3 are applied to compute the correlations involving the total discounted dividends until ruin $D_{\delta_{2}}\left(\tau_{b}\right)$, the aggregate discounted claim amount until ruin $\sum_{k=1}^{N\left(\tau_{b}\right)} e^{-\delta_{3} T_{k}} Y_{k}$, and the time of ruin $\tau_{b}$ (which is considered only when ruin occurs). Hence, we assume a cost function of $f(y)=y$ (i.e., $Z_{\delta_{3}}\left(\tau_{b}\right)=\sum_{k=1}^{N\left(\tau_{b}\right)} e^{-\delta_{3} T_{k}} Y_{k}$ ) and a penalty of $w \equiv 1$ throughout this section. With initial surplus $U_{b}(0)=u$ and threshold level $b$, for notational convenience we denote the unconditional expectation of a random variable $X$ by $E[X \mid u ; b]$, and we shall use $E_{r}[X \mid u ; b]\left(E_{s}[X \mid u ; b]\right.$, respectively) to denote the expectation of $X$ conditional on the event $\left\{\tau_{b}<\infty\right\}\left(\left\{\tau_{b}=\infty\right\}\right.$, respectively). The subscripts " $r$ " and "s" correspond to "ruin" and "survival", respectively. Clearly, one has

$$
E_{r}[X \mid u ; b]=\frac{E\left[X 1_{\left\{\tau_{b}<\infty\right\}} \mid u ; b\right]}{\psi(u ; b)}
$$


and

$$
E_{s}[X \mid u ; b]=\frac{E\left[X 1_{\left\{\tau_{b}=\infty\right\}} \mid u ; b\right]}{\varphi(u ; b)}
$$

where $\psi(u ; b)$ and $\varphi(u ; b)$ are the ruin probability and survival probability, respectively. Then the correlation of the random variables $X_{1}$ and $X_{2}$ is given by

$$
\operatorname{Corr}_{\bullet}\left(X_{1}, X_{2} \mid u ; b\right)=\frac{\operatorname{Cov}_{\bullet}\left(X_{1}, X_{2} \mid u ; b\right)}{\sqrt{\operatorname{Var}_{\bullet}\left(X_{1} \mid u ; b\right) \operatorname{Var}_{\bullet}\left(X_{2} \mid u ; b\right)}}
$$

where

$$
\operatorname{Cov} \bullet\left(X_{1}, X_{2} \mid u ; b\right)=E_{\bullet}\left[X_{1} X_{2} \mid u ; b\right]-E_{\bullet}\left[X_{1} \mid u ; b\right] E_{\bullet}\left[X_{2} \mid u ; b\right]
$$

is the covariance of $X_{1}$ and $X_{2}$, and

$$
\operatorname{Var}_{\bullet}(X \mid u ; b)=E_{\bullet}\left[X^{2} \mid u ; b\right]-\left(E_{\bullet}[X \mid u ; b]\right)^{2}
$$

is the variance of $X$. Here the expectation $E_{\mathbf{e}}$ can be the unconditional expectation $E$ or the conditional expectations $E_{r}$ or $E_{s}$, and this applies to other moment-based quantities as well. All intermediate quantities required in our computation involving $\tau_{b}, D_{\delta_{2}}\left(\tau_{b}\right)$ and $Z_{\delta_{3}}\left(\tau_{b}\right)$ are obtainable from the Gerber-Shiu function $\phi_{\delta_{1}, \delta_{2}, \delta_{3}, n, m}(u ; b)=\phi_{\delta_{123}, n, m}(u ; b)$ in $(1.5)$ and the joint moment $\varphi_{\delta_{2}, \delta_{3}, n, m}(u ; b)=$ $\varphi_{\delta_{23}, n, m}(u ; b)$ in (1.6). For example, the first joint moment $E_{r}\left[D_{\delta_{2}}\left(\tau_{b}\right) Z_{\delta_{3}}\left(\tau_{b}\right) \mid u ; b\right]$ conditional on ruin is the ratio of $E\left[D_{\delta_{2}}\left(\tau_{b}\right) Z_{\delta_{3}}\left(\tau_{b}\right) 1_{\left\{\tau_{b}<\infty\right\}} \mid u ; b\right]=\left.\phi_{\delta_{123}, 1,1}(u ; b)\right|_{\delta_{1}=0}$ to $\psi(u ; b)=\left.\phi_{\delta_{1}}(u ; b)\right|_{\delta_{1}=0}$, which can be evaluated using Theorem 10 and Lemma 4. Similarly, the first joint moment $E_{s}\left[D_{\delta_{2}}\left(\tau_{b}\right) Z_{\delta_{3}}\left(\tau_{b}\right) \mid u ; b\right]$ conditional on survival follows from $E\left[D_{\delta_{2}}\left(\tau_{b}\right) Z_{\delta_{3}}\left(\tau_{b}\right) 1_{\left\{\tau_{b}=\infty\right\}} \mid u ; b\right]=$ $\varphi_{\delta_{23}, 1,1}(u ; b)$ (that is available in Theorem 11) and $\varphi(u ; b)=1-\psi(u ; b)$. The unconditional first joint moment $E\left[D_{\delta_{2}}\left(\tau_{b}\right) Z_{\delta_{3}}\left(\tau_{b}\right) \mid u ; b\right]$ is given by (1.7) when $n=m=1$. It is instructive to note that correlations in relation to $\tau_{b}$ only exist when $\tau_{b}<\infty$, and the (joint) moments involving $\tau_{b}$ can be obtained from $\phi_{\delta_{123}, n, m}(u ; b)$. For example, one has

$$
E_{r}\left[\tau_{b} Z_{\delta_{3}}\left(\tau_{b}\right) \mid u ; b\right]=\frac{E\left[\tau_{b} Z_{\delta_{3}}\left(\tau_{b}\right) 1_{\left\{\tau_{b}<\infty\right\}} \mid u ; b\right]}{\psi(u ; b)}=-\frac{\left.\frac{\partial}{\partial \delta_{1}} \phi_{\delta_{123}, 0,1}(u ; b)\right|_{\delta_{1}=0}}{\psi(u ; b)}
$$

Before discussing specific examples, we first note that in general the relationship

$$
u+c \tau_{b}+\left|U_{b}\left(\tau_{b}\right)\right|=D\left(\tau_{b}\right)+S\left(\tau_{b}\right) \text { on the set }\left\{\tau_{b}<\infty\right\}
$$

among the random variables is valid for sample paths leading to ruin, where $D\left(\tau_{b}\right)$ is the total dividends paid until ruin and $S\left(\tau_{b}\right)=\sum_{k=1}^{N\left(\tau_{b}\right)} Y_{k}$ is the aggregate claims until ruin (both without discounting). For these sample paths, it is clear that

$$
D\left(\tau_{b}\right) \leq \alpha \tau_{b} \text { on the set }\left\{\tau_{b}<\infty\right\}
$$

and therefore

$$
S\left(\tau_{b}\right) \geq u+c_{2} \tau_{b}+\left|U_{b}\left(\tau_{b}\right)\right| \text { on the set }\left\{\tau_{b}<\infty\right\}
$$

On the other hand, concerning the sample paths for which the process survives, one has that

$$
D(t)+S(t) \leq u+c t \text { for all } t \geq 0 \text { on the } \operatorname{set}\left\{\tau_{b}=\infty\right\}
$$


The parameter values that are used for all numerical illustrations are summarised in Table 1 . In each subsequent figure, the quantity of interest is plotted against the initial surplus level $u$ under three different claim size distributions, namely

i. a sum of two exponentials ("Sum Exp") with density $p(y)=3 e^{-(3 / 2) y}-3 e^{-3 y}$;

ii. an exponential distribution ("Exp") with density $p(y)=e^{-y}$; and

iii. a mixture of two exponentials ("Mixed Exp") with density $p(y)=(1 / 6) e^{-(1 / 2) y}+(4 / 3) e^{-2 y}$.

All these distributions belong to the class of combinations of exponentials (see (3.1)) and have the same mean of 1 (and the loading condition $c_{2}>\lambda E\left[Y_{1}\right]$ holds true). However, they have different amount of variability as evident in their variances of $0.56,1$, and 2 , respectively. The curves corresponding to the above claim distributions are marked in solid, dashed, and dotted lines, respectively.

Conditional on ruin occurring, Figures $1-3$ show how the pairwise correlations of $\tau_{10}$ and $Z_{0.01}\left(\tau_{10}\right)$ and $D_{0.01}\left(\tau_{10}\right)$ vary with $u$ for $0 \leq u \leq 200$. (For simplicity, we shall write $\tau, Z$, and $D$ instead of $\tau_{10}$, $Z_{0.01}\left(\tau_{10}\right)$, and $D_{0.01}\left(\tau_{10}\right)$, respectively, in the $y$-axis of the plots.) From Figure 1 , it is observed that $\operatorname{Corr}_{r}\left(\tau_{10}, Z_{0.01}\left(\tau_{10}\right) \mid u ; 10\right)$ for all three claim distributions is of the same shape. Specifically, $\operatorname{Corr}_{r}\left(\tau_{10}, Z_{0.01}\left(\tau_{10}\right) \mid u ; 10\right)$ starts with a positive value of over 0.9 , and it decreases as $u$ increases and then becomes negative when $u$ reaches approximately 95. This complements figures 1 and 2 in Cheung \& Woo (2016), which demonstrated a sign change of the covariance of the ruin time and the aggregate discounted claims until ruin in a dependent Sparre Andersen risk model without dividends as $u$ increases. Some interpretations therein are indeed applicable: for fixed $u$, two opposing effects are in place when one analyses sample paths for which $\tau_{b}$ is large. Intuitively, the aggregate (non-discounted)

Table 1. Parameters used in all numerical examples.

\begin{tabular}{lc}
\hline \hline Parameters & Value \\
\hline Threshold level $b$ & 10 \\
Premium rate $c=c_{1}$ & 1.5 \\
Dividend rate $\alpha=c_{1}=c_{2}$ & 0.2 \\
Poisson arrival rate $\lambda$ & 1 \\
Shareholders' force of interest $\delta_{2}$ & 0.01 \\
Policyholders' force of interest $\delta_{3}$ & 0.01 \\
\hline \hline
\end{tabular}

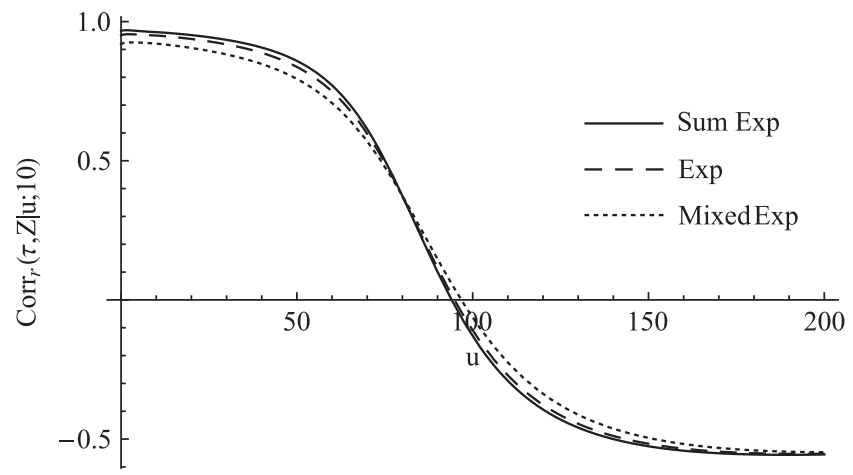

Figure 1. Correlation of ruin time and aggregate discounted claims conditional on ruin. 


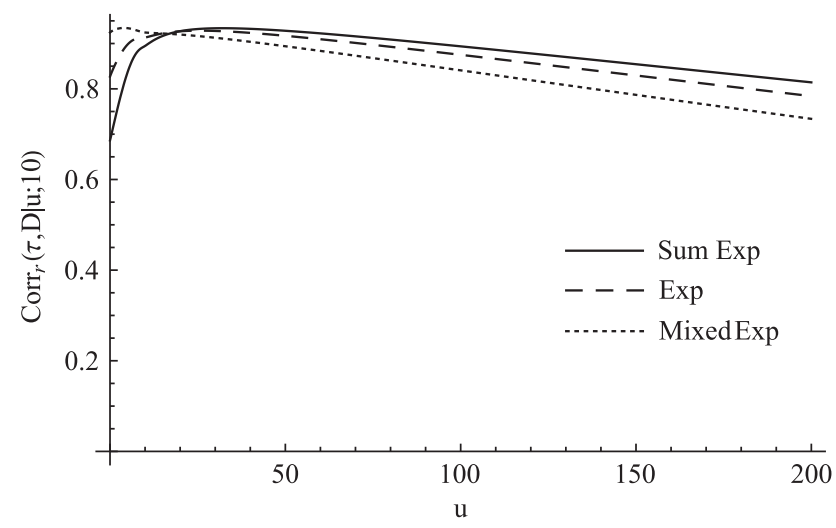

Figure 2. Correlation of ruin time and discounted dividends conditional on ruin.

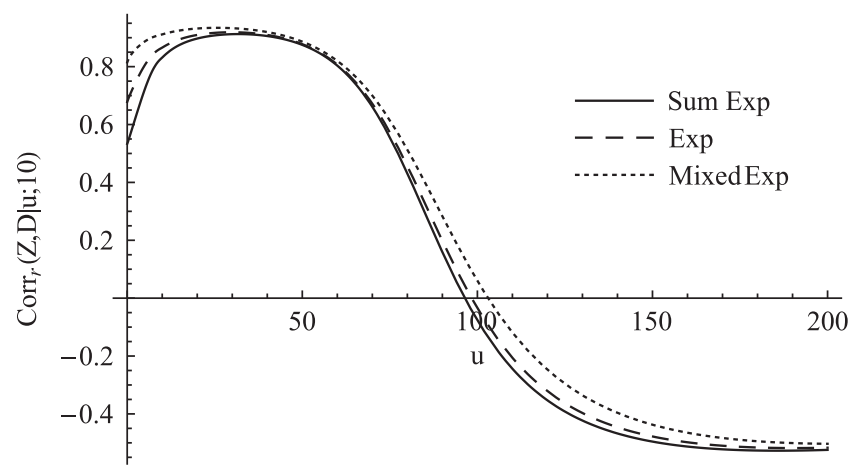

Figure 3. Correlation of aggregate discounted claims and discounted dividends conditional on ruin.

claim amount $S\left(\tau_{10}\right)=\sum_{k=1}^{N\left(\tau_{10}\right)} Y_{k}$ tends to be large because more claims arise as the process survives longer (see also (4.3)). But these claims occur over a longer time horizon and a large claim does not happen early (otherwise it would have caused early ruin), meaning that the discounted amount $Z_{0.01}\left(\tau_{10}\right)=\sum_{k=1}^{N\left(\tau_{10}\right)} e^{-0.01 T_{k}} Y_{k}$ possibly has a tendency to become smaller due to discounting. Figure 1 suggests that the former effect is more dominant until the correlation changes sign at around $u=95$. As $u$ increases further from 95, the effect of discounting starts to dominate because the discounting on the nominal amount $u$ appearing on the right-hand side of $(4.3)$ is getting significant.

Next, when we look at Figure 2, which depicts the behaviour of $\operatorname{Corr}_{r}\left(\tau_{10}, D_{0.01}\left(\tau_{10}\right) \mid u ; 10\right)$, it is noted that the correlation is always positive. This is unlike $\operatorname{Corr}_{r}\left(\tau_{10}, Z_{0.01}\left(\tau_{10}\right) \mid u ; 10\right)$ in Figure 1 where there is a change in sign as $u$ increases. A possible explanation is that $u$ does not appear on the right-hand side of (4.2) (as dividend is paid from part of the premium income but not the initial surplus), and thus the effect of discounting on $u$ is absent in this case. As a result, the positive correlation between $\tau_{10}$ and $D_{0.01}\left(\tau_{10}\right)$ is simply attributed to the fact that, for each fixed $u$, the surplus process is more likely to stay above the threshold more often when the ruin time is large, resulting in more dividends.

In Figure 3, the correlation $\operatorname{Corr}_{r}\left(Z_{0.01}\left(\tau_{10}\right), D_{0.01}\left(\tau_{10}\right) \mid u ; 10\right)$ conditional on ruin takes on positive values when $u$ increases to about 100 and then it becomes negative. From the above discussions, we 
argue that the aggregate non-discounted values of the claim amount $S\left(\tau_{10}\right)$ and dividends $D\left(\tau_{10}\right)$ both tend to increase with the ruin time $\tau_{10}$. However, $S\left(\tau_{10}\right)$ and $D\left(\tau_{10}\right)$ may also move in opposite directions because only part of the claims are paid from the premium, while all dividend payments come from the premium (see also (4.1) for the constraint on the sum $D\left(\tau_{10}\right)+S\left(\tau_{10}\right)$ ). Under discounting, Figure 3 suggests that the former effect dominates for $u$ less than 100, while the latter becomes dominant when $u$ exceeds 100. Interestingly, we observe that $\operatorname{Corr}_{r}\left(Z_{0.01}\left(\tau_{10}\right), D_{0.01}\left(\tau_{10}\right) \mid u ; 10\right)$ in Figure 3 changes sign at roughly the same place as $\operatorname{Corr}_{r}\left(\tau_{10}, Z_{0.01}\left(\tau_{10}\right) \mid u ; 10\right)$ does in Figure 1. Note also that the curves in Figure 3 are ordered according to the variance of the individual claim size distribution.

Now, we turn to Figure 4 concerning the correlation of $Z_{0.01}\left(\tau_{10}\right)$ and $D_{0.01}\left(\tau_{10}\right)$ conditional on survival. Clearly, the shape of $\operatorname{Corr}_{s}\left(Z_{0.01}\left(\tau_{10}\right), D_{0.01}\left(\tau_{10}\right) \mid u ; 10\right)$ is completely different from that of $\operatorname{Corr}_{r}\left(Z_{0.01}\left(\tau_{10}\right), D_{0.01}\left(\tau_{10}\right) \mid u ; 10\right)$ in Figure 3. In particular, $\operatorname{Corr}_{s}\left(Z_{0.01}\left(\tau_{10}\right), D_{0.01}\left(\tau_{10}\right) \mid u ; 10\right)$ in Figure 4 begins at a negative value between -0.55 and -0.60 . It increases with $u$, stays negative and converges to 0 from below. Note also that the above pattern appears to kick in earlier when the claim size has smaller variance. The reason for negative correlation is the constraint (4.4), which makes it impossible for both $Z_{0.01}\left(\tau_{10}\right)$ and $D_{0.01}\left(\tau_{10}\right)$ to be large in the presence of discounting. The convergence of $\operatorname{Corr}_{s}\left(Z_{0.01}\left(\tau_{10}\right), D_{0.01}\left(\tau_{10}\right) \mid u ; 10\right)$ to 0 as $u$ increases can be explained by zero covariance at the limit. Indeed, we can apply Lemma 2 three times with $(n, m)=(1,1),(1,0)$, and $(0,1)$ to see that

$$
\begin{aligned}
& \lim _{u \rightarrow \infty} E\left[D_{\delta_{2}}\left(\tau_{b}\right) Z_{\delta_{3}}\left(\tau_{b}\right) 1_{\left\{\tau_{b}=\infty\right\}} \mid u ; b\right] \\
& \quad=\left(\lim _{u \rightarrow \infty} E\left[D_{\delta_{2}}\left(\tau_{b}\right) 1_{\left\{\tau_{b}=\infty\right\}} \mid u ; b\right]\right)\left(\lim _{u \rightarrow \infty} E\left[Z_{\delta_{3}}\left(\tau_{b}\right) 1_{\left\{\tau_{b}=\infty\right\}} \mid u ; b\right]\right)
\end{aligned}
$$

Division of each of the three above limits by the limiting survival probability $\lim _{u \rightarrow \infty} \varphi(u ; b)=1$ reveals that $\operatorname{Cov}_{s}\left(Z_{\delta_{3}}\left(\tau_{b}\right), D_{\delta_{2}}\left(\tau_{b}\right) \mid u ; b\right)=E_{s}\left[Z_{\delta_{3}}\left(\tau_{b}\right) D_{\delta_{2}}\left(\tau_{b}\right) \mid u ; b\right]-E_{s}\left[Z_{\delta_{3}}\left(\tau_{b}\right) \mid u ; b\right] E_{s}\left[D_{\delta_{2}}\left(\tau_{b}\right) \mid u ; b\right]$ tends to 0 as $u \rightarrow \infty$.

Lastly, Figure 5 plots the unconditional correlation of $Z_{0.01}\left(\tau_{10}\right)$ and $D_{0.01}\left(\tau_{10}\right)$ against $u$. As a function of $u$, the correlation $\operatorname{Corr}\left(Z_{0.01}\left(\tau_{10}\right), D_{0.01}\left(\tau_{10}\right) \mid u ; 10\right)$ first decreases from over 0.95 to negative values and finally converges to 0 . Similar to Figure 4 , the pattern prevails earlier when the individual claim size has less variability. Note that the unconditional correlation takes all sample

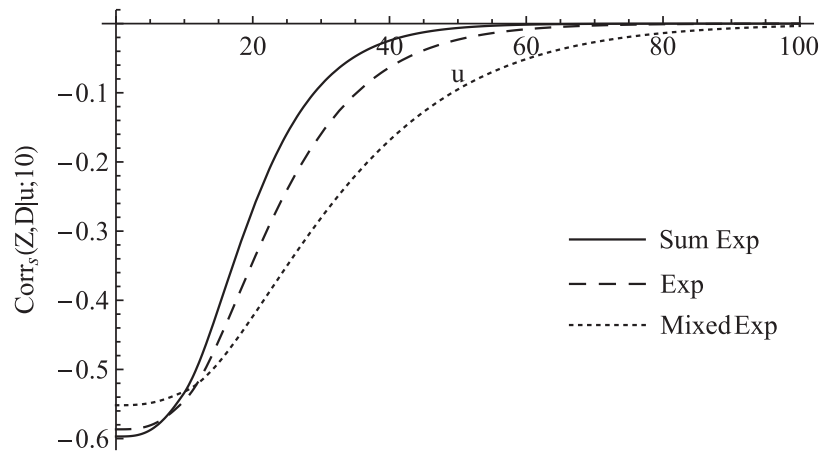

Figure 4. Correlation of aggregate discounted claims and discounted dividends conditional on survival. 


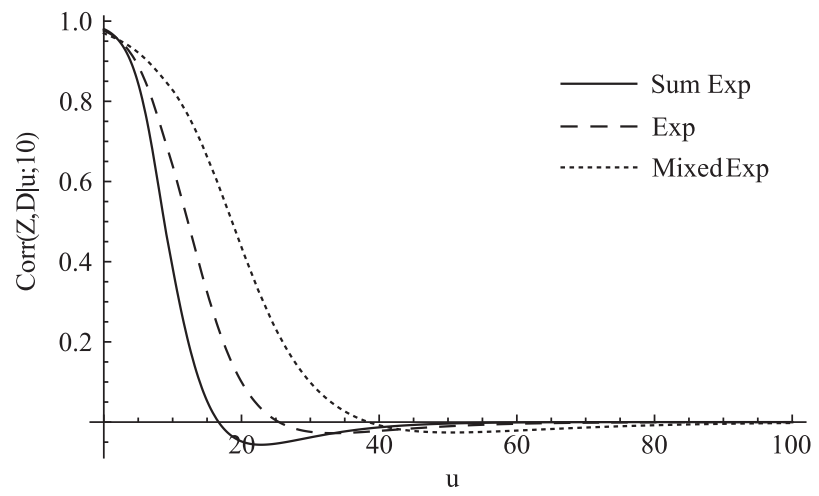

Figure 5. Unconditional correlation of aggregate discounted claims and discounted dividends.

paths into account regardless of whether the process ruins or survives. The contributions of these events are in accordance with the ruin probability $\psi(u ; 10)$ and the survival probability $\varphi(u ; 10)=1-\psi(u ; 10)$, respectively. As $u$ increases, $\psi(u ; 10)$ decreases and converges to 0 and therefore the impact of ruin occurrence becomes less significant while that of survival becomes stronger. The shape of Figure 5 is thus a combination of Figures 3 and 4.

\section{Concluding Remarks}

This paper jointly analyses the aggregate discounted claims until ruin (payments to policyholders) and the discounted dividends until ruin (payments to shareholders) in the compound Poisson insurance risk model with a threshold dividend strategy. The method adopted here is based on the derivation of recursive IDEs satisfied by Gerber-Shiu-type functions involving the joint moments of these two random variables. When each claim amount is distributed as a combination of exponentials, these IDEs are transformed to ordinary differential equations that can be solved with the help of the continuity and limiting conditions.

An alternative approach will be to connect the risk process to an equivalent fluid flow model (e.g., Badescu et al., 2005). In such a construction, a downward jump (caused by the arrival of a claim) of size $y$ in the risk model is replaced by decreasing segment of slope $-C$ in the fluid model over a time period of $y / C$ for some constant $C>0$. It is well known that (e.g., Ramaswami, 2006; Ahn et al., 2007) the Laplace transforms of various first passage times can typically be expressed in terms of the Laplace transform of the busy period, whose evaluation can be done by numerical algorithms that converge quadratically fast (e.g., Ahn \& Ramaswami, 2005; Bean et al., 2005). Then one may try to express our Gerber-Shiu-type functions in terms of these quantities pertaining to the fluid model. While the moments of discounted dividends were derived by Badescu \& Landriault (2008) in this manner for a multi-threshold model, the aggregate discounted claim amount until ruin has never been analysed via fluid flow to the best of our knowledge. Nevertheless, in the absence of discounting, we note that the total dividend is (a scalar multiple of) the occupation time when the fluid level is in an increasing phase above the threshold level $b$, while the aggregate claim amount corresponds to (a scalar multiple of) the occupation time of the fluid in a decreasing phase. Finally, we also remark that another research problem will be to determine the exact joint distribution of the discounted dividends and the aggregate discounted claims until ruin (as opposed to joint moments in the present work), which is expected to be a very challenging task. We leave these as open questions. 


\section{Acknowledgements}

The authors would like to thank the anonymous reviewers for their helpful comments and suggestions which improved both the content and the presentation of an earlier version of the paper. E. C. K. Cheung acknowledges the support from the Research Grants Council of the Hong Kong Special Administrative Region (Project No. HKU 701212P). This research is also partially supported by the CAE 2013 research grant from the Society of Actuaries (SOA). Any opinions, findings, and conclusions or recommendations expressed in this material are those of the authors and do not necessarily reflect the views of the SOA.

\section{References}

Ahn, S., Badescu, A.L. \& Ramaswami, V. (2007). Time dependent analysis of finite buffer fluid flows and risk models with a dividend barrier. Queueing Systems, 55(4), 207-222.

Ahn, S. \& Ramaswami, V. (2005). Efficient algorithms for transient analysis of stochastic fluid flow models. Journal of Applied Probability, 42(2), 531-549.

Albrecher, H. \& Hartinger, J. (2007). A risk model with multilayer dividend strategy. North American Actuarial Journal, 11(2), 43-64.

Albrecher, H., Hartinger, J. \& Thonhauser, S. (2007). On exact solutions for dividend strategies of threshold and linear barrier type in a Sparre Andersen model. ASTIN Bulletin, 37(2), 203-233.

Albrecher, H. \& Thonhauser, S. (2009). Optimality results for dividend problems in insurance. Revista de la Real Academia de Ciencias Exactas, Fisicas y Naturales Serie A. Matematicas, 103(2), 295-320.

Avanzi, B. (2009). Strategies for dividend distribution: a review. North American Actuarial Journal, 13(2), 217-251.

Badescu, A., Breuer, L., Da Silva Soares, A., Latouche, G., Remiche, M.-A. \& Stanford, D. (2005). Risk processes analyzed as fluid queues. Scandinavian Actuarial Journal, 2005(2), 127-141.

Badescu, A.L., Drekic, S. \& Landriault, D. (2007a). Analysis of a threshold dividend strategy for a MAP risk model. Scandinavian Actuarial Journal, 2007(4), 227-247.

Badescu, A.L., Drekic, S. \& Landriault, D. (2007b). On the analysis of a multi-threshold Markovian risk model. Scandinavian Actuarial Journal, 2007(4), 248-260.

Badescu, A.L. \& Landriault, D. (2008). Recursive calculation of the dividend moments in a multithreshold risk model. North American Actuarial Journal, 12(1), 74-88.

Bean, N.G., O'Reilly, M.M. \& Taylor, P.G. (2005). Hitting probabilities and hitting times for stochastic fluid flows. Stochastic Processes and their Applications, 115(9), 1530-1556.

Cai, J., Feng, R. \& Willmot, G.E. (2009). On the expectation of total discounted operating costs up to default and its applications. Advances in Applied Probability, 41(2), 495-522.

Cheung, E.C.K. (2013). Moments of discounted aggregate claim costs until ruin in a Sparre Andersen risk model with general interclaim times. Insurance: Mathematics and Economics, 53(2), 343-354.

Cheung, E.C.K., Dickson, D.C.M. \& Drekic, S. (2008). Moments of discounted dividends for a threshold strategy in the compound Poisson risk model. North American Actuarial Journal, 12(3), 299-318.

Cheung, E.C.K. \& Feng, R. (2013). A unified analysis of claim costs up to ruin in a Markovian arrival risk process. Insurance: Mathematics and Economics, 53(1), 98-109.

Cheung, E.C.K., Liu, H. \& Woo, J.-K. (2015). On the joint analysis of the total discounted payments to policyholders and shareholders: dividend barrier strategy. Risks, 3(4), 491-514.

Cheung, E.C.K. \& Woo, J.-K. (2016). On the discounted aggregate claim costs until ruin in dependent Sparre Andersen risk processes. Scandinavian Actuarial Journal, 2016(1), 63-91. 
de Finetti, B. (1957). Su un'impostazione alternativa della teoria collettiva del rischio. Transactions of the XVth International Congress of Actuaries, 2, 433-443.

Dickson, D.C.M. \& Drekic, S. (2006). Optimal dividends under a ruin probability constraint. Annals of Actuarial Science, 1(2), 291-306.

Dickson, D.C.M. \& Hipp, C. (2001). On the time to ruin for $\operatorname{Erlang(2)~risk~processes.~Insurance:~}$ Mathematics and Economics, 29(3), 333-344.

Dufresne, D. (2007). Fitting combinations of exponentials to probability distributions. Applied Stochastic Models in Business and Industry, 23(1), 23-48.

Feng, R. (2009a). On the total operating costs up to default in a renewal risk model. Insurance: Mathematics and Economics, 45(2), 305-314.

Feng, R. (2009b). A matrix operator approach to the analysis of ruin-related quantities in the phase-type renewal risk model. Bulletin of the Swiss Association of Actuaries, 2009(1 and 2), 71-87.

Gerber, H.U. (1979). An Introduction to Mathematical Risk Theory. Huebner Foundation Monograph 8. Richard D. Irwin, Homewood, IL.

Gerber, H.U. \& Shiu, E.S.W. (1998). On the time value of ruin. North American Actuarial Journal, 2(1), 48-72.

Gerber, H.U. \& Shiu, E.S.W. (2006). On optimal dividend strategies in the compound Poisson model. North American Actuarial Journal, 10(2), 76-93.

Gerber, H.U., Shiu, E.S.W. \& Smith, N. (2006). Maximizing dividends without bankruptcy. ASTIN Bulletin, 36(1), 5-23.

Ji, L. \& Zhang, C. (2012). Analysis of the multiple roots of the Lundberg fundamental equation in the $\mathrm{PH}(n)$ risk model. Applied Stochastic Models in Business and Industry, 28(1), 73-90.

Kyprianou, A.E. (2013). Gerber-Shiu Risk Theory. Springer, Cham, Heidelberg, New York, Dordrecht, London.

Kyprianou, A.E. \& Loeffen, R.L. (2010). Refracted Lévy processes. Annales de l'Institut Henri Poincaré - Probabilités et Statistiques, 46(1), 24-44.

Léveillé, G. \& Garrido, J. (2001). Recursive moments of compound renewal sums with discounted claims. Scandinavian Actuarial Journal, 2001(2), 98-110.

Li, S. \& Garrido, J. (2004). On ruin for the $\operatorname{Erlang}(n)$ risk process. Insurance: Mathematics and Economics, 34(3), 391-408.

Lin, X.S. \& Pavlova, K.P. (2006). The compound Poisson risk model with a threshold dividend strategy. Insurance: Mathematics and Economics, 38(1), 57-80.

Lin, X.S. \& Sendova, K.P. (2008). The compound Poisson risk model with multiple thresholds. Insurance: Mathematics and Economics, 42(2), 617-627.

Loeffen, R.L. (2008). On optimality of the barrier strategy in de Finetti's dividend problem for spectrally negative Lévy processes. Annals of Applied Probability, 18(5), 1669-1680.

Lu, Y. \& Li, S. (2009). The Markovian regime-switching risk model with a threshold dividend strategy. Insurance: Mathematics and Economics, 44(2), 296-303.

Mihálykó, É.O. \& Mihálykó, C. (2011). Mathematical investigation of the Gerber-Shiu function in the case of dependent inter-claim time and claim size. Insurance: Mathematics and Economics, 48(3), 378-383.

Ramaswami, V. (2006). Passage times in fluid models with application to risk processes. Methodology and Computing in Applied Probability, 8(4), 497-515.

Taylor, G.C. (1979). Probability of ruin under inflationary conditions or under experience rating. ASTIN Bulletin, 10(2), 149-162.

Willmot, G.E. (1989). The total claims distribution under inflationary conditions. Scandinavian Actuarial Journal, 1989(1), 1-12. 
Woo, J.-K. \& Cheung, E.C.K. (2013). A note on discounted compound renewal sums under dependency. Insurance: Mathematics and Economics, 52(2), 170-179.

Woo, J.-K. \& Liu, H. (2014). Discounted aggregate claim costs until ruin in the discrete-time renewal risk model. Preprint.

Zhou, X. (2004). When does surplus reach a certain level before ruin? Insurance: Mathematics and Economics, 35(3), 553-561.

Zhu, J. \& Yang, H. (2008). Ruin theory for a Markov regime-switching model under a threshold dividend strategy. Insurance: Mathematics and Economics, 42(1), 311-318.

\section{Appendix: Proofs of Theorems and Lemmas in Sections 2 and 3}

Proof of Theorem 1: We use the standard approach of considering the time interval $(0, h]$ for some small $h>0$. Conditioning on all possible events together with a binomial expansion (if a claim occurs) yields, for $0 \leq u<b$

$$
\begin{aligned}
\phi_{\delta_{13}, m}(u ; b)= & (1-\lambda b) e^{-\left(\delta_{1}+m \delta_{3}\right) b} \phi_{\delta_{13}, m}\left(u+c_{1} b ; b\right) \\
& +\lambda h e^{-\left(\delta_{1}+m \delta_{3}\right) b}\left[\int_{0}^{u+c_{1} b} \sum_{i=0}^{m}\left(\begin{array}{c}
m \\
i
\end{array}\right) f^{m-i}(y) \phi_{\delta_{13}, i}\left(u+c_{1} b-y ; b\right) p(y) d y\right. \\
& \left.+\int_{u+c_{1} b}^{\infty} f^{m}(y) w\left(u+c_{1} h, y-u-c_{1} b\right) p(y) d y\right]+o(b)
\end{aligned}
$$

Letting $b \rightarrow 0^{+}$, one observes that $\phi_{\delta_{13}, m}(u ; b)$ is right-continuous in $u$ for $0 \leq u<b$. Since $e^{-\left(\delta_{1}+m \delta_{3}\right) b}=1-\left(\delta_{1}+m \delta_{3}\right) b+o(b)$, rearrangements and division by $h$ give, for $0<u \leq b$

$$
\begin{aligned}
& \frac{\phi_{\delta_{13}, m}\left(u+c_{1} b ; b\right)-\phi_{\delta_{13}, m}(u ; b)}{h}-\left(\lambda+\delta_{1}+m \delta_{3}\right) \phi_{\delta_{13}, m}\left(u+c_{1} b ; b\right) \\
& +\lambda e^{-\left(\delta_{1}+m \delta_{3}\right) b}\left[\int_{0}^{u+c_{1} h} \sum_{i=0}^{m}\left(\begin{array}{c}
m \\
i
\end{array}\right) f^{m-i}(y) \phi_{\delta_{13}, i}\left(u+c_{1} b-y ; b\right) p(y) d y\right. \\
& \left.+\int_{u+c_{1} b}^{\infty} f^{m}(y) w\left(u+c_{1} b, y-u-c_{1} h\right) p(y) d y\right]+\frac{o(b)}{b}=0
\end{aligned}
$$

Again, sending $b \rightarrow 0^{+}$and noting that the above equation only involves $\phi_{\delta_{13}, i}(\cdot ; b)$ in the lower layer, we obtain (2.2) with $\phi_{L, \delta_{13}, m}^{\prime}(u ; b)$ being a right derivative. If we replace $u$ by $u-c_{1} b$ in (A.1), then similar procedure reveals that $\phi_{\delta_{13}, m}(u ; b)$ is left-continuous in $u$ for $0<u \leq b$ and (2.2) also holds true with $\phi_{L, \delta_{13}, m}^{\prime}(u ; b)$ being a left derivative.

For $u \geq b$, it can be easily seen that (A.1) (and hence (A.2)) is also applicable but with $c_{1}$ replaced by $c_{2}$. Therefore, following the same arguments as above, one can conclude that $\phi_{\delta_{13}, m}(u ; b)$ is continuous for $u \geq b$. Further noting that, for $u \geq b$

$$
\begin{aligned}
& \int_{0}^{u} f^{m-i}(y) \phi_{\delta_{13}, i}(u-y ; b) p(y) d y \\
& =\int_{0}^{u-b} f^{m-i}(y) \phi_{U, \delta_{13}, i}(u-y ; b) p(y) d y+\int_{u-b}^{u} f^{m-i}(y) \phi_{L, \delta_{13}, i}(u-y ; b) p(y) d y
\end{aligned}
$$


it is found that (2.3) is valid for both right and left derivatives of $\phi_{U, \delta_{13}, m}(\cdot ; b)$. Finally, the continuity condition (2.4) is a direct consequence of the left-continuity of $\phi_{\delta_{13}, m}(u ; b)$ at $u=b$ in the lower layer and the right-continuity of $\phi_{\delta_{13}, m}(u ; b)$ at $u=b$ in the upper layer.

Proof of Theorem 2: The same method as in the proof of Theorem 1 of considering a small time interval $(0, h]$ can be adopted. If the process $\left\{U_{b}(t)\right\}_{t \geq 0}$ starts below the threshold level $b$, then it is possible that $D_{\delta_{2}}\left(\tau_{b}\right)=0$ (when the process $\left\{U_{b}(t)\right\}_{t \geq 0}$ never reaches $b$ before ruin). We first arrive at, for $0 \leq u<b$

$$
\begin{aligned}
\phi_{\delta_{123}, n, m}(u ; b)= & (1-\lambda h) e^{-\left(\delta_{1}+n \delta_{2}+m \delta_{3}\right) h} \phi_{\delta_{123}, n, m}\left(u+c_{1} b ; b\right) \\
& +\lambda h e^{-\left(\delta_{1}+n \delta_{2}+m \delta_{3}\right) b} \int_{0}^{u+c_{1} h} \sum_{i=0}^{m}\left(\begin{array}{c}
m \\
i
\end{array}\right) f^{m-i}(y) \phi_{\delta_{123}, n, i}\left(u+c_{1} b-y ; b\right) p(y) d y+o(b)(\mathrm{A} .3)
\end{aligned}
$$

The above equation is almost identical to (A.1) in Theorem 1, except that the term $\int_{u+c_{1} b}^{\infty} f^{m}(y) w\left(u+c_{1} b, y-u-c_{1} b\right) p(y) d y$ is now absent. Following the arguments therein, one can see that $\phi_{\delta_{123}, n, m}(u ; b)$ is continuous in $u$ for $0 \leq u \leq b$, and (2.5) holds true.

On the other hand, if $u \geq b$, dividends are paid continuously at rate $\alpha$ until the surplus falls below $b$. Denoting $\bar{s}_{\bar{t} \delta}=\left(e^{\delta t}-1\right) / \delta$ as the actuarial symbol for the accumulated value of an annuity with rate $\$ 1$ per unit time payable continuously for $t$ time units under a force of interest $\delta$, we have that, for $u \geq b$

$$
\begin{aligned}
\phi_{\delta_{123}, n, m}(u ; b)= & (1-\lambda b) e^{-\left(\delta_{1}+n \delta_{2}+m \delta_{3}\right) b} \sum_{j=0}^{n}\left(\begin{array}{c}
n \\
j
\end{array}\right)\left(\alpha \bar{s}_{\bar{b} \mid \delta_{2}}\right)^{j} \phi_{\delta_{123}, n-j, m}\left(u+c_{2} b ; b\right) \\
& +\lambda h e^{-\left(\delta_{1}+n \delta_{2}+m \delta_{3}\right) b}\left[\int_{0}^{u+c_{2} b} \sum_{j=0}^{n}\left(\begin{array}{c}
n \\
j
\end{array}\right)\left(\alpha \bar{s}_{\bar{b} \mid \delta_{2}}\right)^{j} \sum_{i=0}^{m}\left(\begin{array}{c}
m \\
i
\end{array}\right) f^{m-i}(y) \phi_{\delta_{123}, n-j, i}\left(u+c_{2} b-y ; b\right) p(y) d y\right. \\
& \left.+\int_{u+c_{2} b}^{\infty}\left(\alpha \bar{s}_{\bar{b} \mid \delta_{2}}\right)^{n} f^{m}(y) w\left(u+c_{2} h, y-u-c_{2} b\right) p(y) d y\right]+o(b)
\end{aligned}
$$

Noting $\lim _{b \rightarrow 0^{+}} \bar{s}_{\bar{b} \mid \delta_{2}}=0$ and the convention $0^{0}=1$, we separate the contribution $j=0$ in the first summation term above and let $h \rightarrow 0^{+}$to establish the right-continuity of $\phi_{\delta_{123}, n, m}(u ; b)$ for $u \geq b$. As $e^{-\left(\delta_{1}+n \delta_{2}+m \delta_{3}\right) b}=1-\left(\delta_{1}+n \delta_{2}+m \delta_{3}\right) b+o(b)$, rearranging (A.4) and then dividing by $b$ yields, for $u \geq b$

$$
\begin{aligned}
& \frac{\phi_{\delta_{123}, n, m}\left(u+c_{2} h ; b\right)-\phi_{\delta_{123}, n, m}(u ; b)}{h}+\sum_{j=1}^{n}\left(\begin{array}{c}
n \\
j
\end{array}\right) \frac{\left(\alpha \bar{s}_{\bar{b} \mid \delta_{2}}\right)^{j}}{h} \phi_{\delta_{123}, n-j, m}\left(u+c_{2} b ; b\right) \\
& -\left(\lambda+\delta_{1}+n \delta_{2}+m \delta_{3}\right) \sum_{j=0}^{n}\left(\begin{array}{c}
n \\
j
\end{array}\right)\left(\alpha \bar{s}_{\bar{b} \mid \delta_{2}}\right)^{j} \phi_{\delta_{123}, n-j, m}\left(u+c_{2} b ; b\right) \\
& +\lambda e^{-\left(\delta_{1}+n \delta_{2}+m \delta_{3}\right) b}\left[\int_{0}^{u+c_{2} h} \sum_{j=0}^{n}\left(\begin{array}{c}
n \\
j
\end{array}\right)\left(\alpha \bar{s}_{\bar{b} \mid \delta_{2}}\right)^{j} \sum_{i=0}^{m}\left(\begin{array}{c}
m \\
i
\end{array}\right) f^{m-i}(y) \phi_{\delta_{123}, n-j, i}\left(u+c_{2} b-y ; b\right) p(y) d y\right. \\
& \left.+\int_{u+c_{2} b}^{\infty}\left(\alpha \bar{s}_{\bar{b} \mid \delta_{2}}\right)^{n} f^{m}(y) w\left(u+c_{2} h, y-u-c_{2} h\right) p(y) d y\right]+\frac{o(b)}{b}=0
\end{aligned}
$$


Because $\lim _{h \rightarrow 0^{+}} \bar{s}_{\bar{b} \mid \delta_{2}} / h=1$ and $\lim _{h \rightarrow 0^{+}}\left(\bar{s}_{\bar{b} \mid \delta_{2}}\right)^{j} / h=0$ for $j>1$, taking the limit $h \rightarrow 0^{+}$in the above equation leads to the IDE (2.6), with $\phi_{U, \delta_{123}, n, m}^{\prime}(u ; b)$ understood to be a right derivative. Next, for $u>b$, by assuming an initial surplus level of $u-c_{2} b$ instead in (A.4), we obtain the left-continuity of $\phi_{U, \delta_{123}, n, m}(u ; b)$ and that $(2.6)$ is valid with $\phi_{U, \delta_{123}, n, m}^{\prime}(u ; b)$ being a left derivative as well. Lastly, the continuity condition (2.7) follows in the same manner as in the proof of Theorem 1.

Proof of Theorem 3: As the definition (1.6) contains the indicator $1_{\left\{\tau_{b}=\infty\right\}}$, sample paths for which a claim amount exceeds the surplus level just before its occurrence contribute nothing to $\varphi_{\delta_{23}, n, m}(u ; b)$. Again, by conditioning on the possible claim events within $(0, h]$ for some small $h$, we have, for $0 \leq u<b$

$$
\begin{aligned}
\varphi_{\delta_{23}, n, m}(u ; b)= & (1-\lambda b) e^{-\left(n \delta_{2}+m \delta_{3}\right) b} \varphi_{\delta_{23}, n, m}\left(u+c_{1} b ; b\right) \\
& +\lambda h e^{-\left(n \delta_{2}+m \delta_{3}\right) b} \int_{0}^{u+c_{1} b} \sum_{i=0}^{m}\left(\begin{array}{c}
m \\
i
\end{array}\right) f^{m-i}(y) \varphi_{\delta_{23}, n, i}\left(u+c_{1} b-y ; b\right) p(y) d y+o(b)
\end{aligned}
$$

and, for $u \geq b$

$$
\begin{aligned}
\varphi_{\delta_{23}, n, m}(u ; b)= & (1-\lambda b) e^{-\left(n \delta_{2}+m \delta_{3}\right) b} \sum_{j=0}^{n}\left(\begin{array}{c}
n \\
j
\end{array}\right)\left(\alpha \bar{s}_{\bar{b} \mid \delta_{2}}\right)^{j} \varphi_{\delta_{23}, n-j, m}\left(u+c_{2} b ; b\right) \\
& +\lambda h e^{-\left(n \delta_{2}+m \delta_{3}\right) b} \int_{0}^{u+c_{2} h} \sum_{j=0}^{n}\left(\begin{array}{c}
n \\
j
\end{array}\right)\left(\alpha \bar{s}_{\bar{b} \mid \delta_{2}}\right)^{j} \sum_{i=0}^{m}\left(\begin{array}{c}
m \\
i
\end{array}\right) f^{m-i}(y) \\
& \times \varphi_{\delta_{23, n}-j, i}\left(u+c_{2} b-y ; b\right) p(y) d y+o(b)
\end{aligned}
$$

While (A.5) is structurally identical to (A.3), (A.6) is like (A.4) except that the term with the penalty function is absent. Hence, the IDEs (2.8) and (2.9) together with the continuity condition (2.10) follow in the same manner as how Theorem 2 (and also Theorem 1) is proved.

Proof of Lemma 2: For presentation purposes, in this proof we shall specify the dependence of the time of ruin on the initial surplus $u$ by writing $\tau_{u, b}$ instead of $\tau_{b}$. Moreover, we let $D_{\delta_{2}}(u, b, t)=$ $\alpha \int_{0}^{t} e^{-\delta_{2} s} 1_{\left\{U_{b}(s) \geq b\right\}} d s$ be the total discounted dividends paid until time $t$. Then, it is clear that $D_{\delta_{2}}(u, b, t)$ is increasing (i.e., non-decreasing) in both $u$ and $t$, and the dividend variable defined via (1.2) shall be written as $D_{\delta_{2}}\left(\tau_{b}\right)=D_{\delta_{2}}\left(u, b, \tau_{u, b}\right)$. In contrast, with $Z_{\delta_{3}}(t)=\sum_{k=1}^{N(t)} e^{-\delta_{3} T_{k}} f\left(Y_{k}\right)$ being the aggregate discounted claim costs until time $t$, it is noted that $Z_{\delta_{3}}\left(\tau_{u, b}\right)=\sum_{k=1}^{N\left(\tau_{u, b}\right)} e^{-\delta_{3} T_{k}} f\left(Y_{k}\right)$ depends on $u$ only via the ruin time $\tau_{u, b}$. In this proof, the initial condition $U_{b}(0)=u$ will be omitted in related expectations and probabilities.

Note that the ruin probability $\psi(u ; b)=\operatorname{Pr}\left\{\tau_{u, b}<\infty\right\}$ is bounded by $\psi(u ; 0)$ for which the net premium income is always $c_{2}$. Under the loading condition $c_{2}>\lambda E\left[Y_{1}\right]$, it is known from e.g. Kyprianou (2013, theorem 4.3 and equation (9.16)) that $\lim _{u \rightarrow \infty} \psi(u ; 0)=0$. Therefore, one has that $\lim _{u \rightarrow \infty} \psi(u ; b)=0$, or equivalently $\lim _{u \rightarrow \infty} \operatorname{Pr}\left\{\tau_{u, b}=\infty\right\}=1$. Meanwhile, for every realisation of the aggregate claims process $\{S(t)\}_{t \geq 0}$, it is clear that $\tau_{u, b}$ is increasing in $u$ and the sequence of sets $\left\{\left\{\tau_{u, b}=\infty\right\}\right\}_{u=1}^{\infty}$ is increasing as well. This leads to $\lim _{u \rightarrow \infty} \operatorname{Pr}\left\{\tau_{u, b}=\infty\right\}=\operatorname{Pr}\left\{\lim _{u \rightarrow \infty}\left\{\tau_{u, b}=\infty\right\}\right\}$. Combining the above two results, we arrive at $\operatorname{Pr}\left\{\lim _{u \rightarrow \infty}\left\{\tau_{u, b}=\infty\right\}\right\}=1$, i.e. the event $\lim _{u \rightarrow \infty}\left\{\tau_{u, b}=\infty\right\}$ occurs a.s.. 
In other words, $1_{\lim _{u \rightarrow \infty}\left\{\tau_{u, b}=\infty\right\}}=1$ a.s.. Again, due to the fact that the set $\left\{\tau_{u, b}=\infty\right\}$ is increasing in $u$, we have $1_{\lim _{u \rightarrow \infty}\left\{\tau_{u, b}=\infty\right\}}=\lim _{u \rightarrow \infty} 1_{\left\{\tau_{u, b}=\infty\right\}}$, and the intermediate result

$$
\lim _{u \rightarrow \infty} 1_{\left\{\tau_{u, b}=\infty\right\}}=1 \text { a.s. }
$$

follows. Next, we look at the limit of (1.6), namely

$$
\lim _{u \rightarrow \infty} \varphi_{\delta_{23}, n, m}(u ; b)=\lim _{u \rightarrow \infty} E\left[D_{\delta_{2}}^{n}\left(u, b, \tau_{u, b}\right) Z_{\delta_{3}}^{m}\left(\tau_{u, b}\right) 1_{\left\{\tau_{u, b}=\infty\right\}}\right]
$$

where $n, m \in \mathbb{N}$. For any sample path of $\{S(t)\}_{t \geq 0}$, it is observed that the random variables $D_{\delta_{2}}\left(u, b, \tau_{u, b}\right), Z_{\delta_{3}}\left(\tau_{u, b}\right)$ and $1_{\left\{\tau_{u, b}=\infty\right\}}$ are all non-negative and increasing in $u$. Applying the Monotone Convergence Theorem to change the order of limit and expectation leads to

$$
\lim _{u \rightarrow \infty} \varphi_{\delta_{23}, n, m}(u ; b)=E\left[\lim _{u \rightarrow \infty}\left(D_{\delta_{2}}^{n}\left(u, b, \tau_{u, b}\right) Z_{\delta_{3}}^{m}\left(\tau_{u, b}\right) 1_{\left\{\tau_{u, b}=\infty\right\}}\right)\right]
$$

Because $D_{\delta_{2}}^{n}\left(u, b, \tau_{u, b}\right) Z_{\delta_{3}}^{m}\left(\tau_{u, b}\right)=D_{\delta_{2}}^{n}(u, b, \infty) Z_{\delta_{3}}^{m}(\infty)$ on the set $\left\{\tau_{u, b}=\infty\right\}$, the above equation can be rewritten as

$$
\lim _{u \rightarrow \infty} \varphi_{\delta_{23}, n, m}(u ; b)=E\left[\lim _{u \rightarrow \infty}\left(D_{\delta_{2}}^{n}(u, b, \infty) Z_{\delta_{3}}^{m}(\infty) 1_{\left\{\tau_{u, b}=\infty\right\}}\right)\right]
$$

Since $N(t) \rightarrow \infty$ a.s. as $t \rightarrow \infty$, we note that $Z_{\delta_{3}}(\infty)=\sum_{k=1}^{\infty} e^{-\delta_{3} T_{k}} f\left(Y_{k}\right)$ a.s. which does not depend on $u$, and therefore

$$
\begin{aligned}
\lim _{u \rightarrow \infty} \varphi_{\delta_{23}, n, m}(u ; b) & =E\left[Z_{\delta_{3}}^{m}(\infty) \lim _{u \rightarrow \infty}\left(D_{\delta_{2}}^{n}(u, b, \infty) 1_{\left\{\tau_{u, b}=\infty\right\}}\right)\right] \\
& =E\left[Z_{\delta_{3}}^{m}(\infty)\left(\lim _{u \rightarrow \infty} D_{\delta_{2}}^{n}(u, b, \infty)\right)\left(\lim _{u \rightarrow \infty} 1_{\left\{\tau_{u, b}=\infty\right\}}\right)\right] \\
& =E\left[Z_{\delta_{3}}^{m}(\infty) \lim _{u \rightarrow \infty} D_{\delta_{2}}^{n}(u, b, \infty)\right]
\end{aligned}
$$

In the second equality above, we have used the fact that the limit of product is the product of limits as long as the individual limits exist. While the limit of $1_{\left\{\tau_{u, b}=\infty\right\}}$ is given by (A.7), the limit $\lim _{u \rightarrow \infty} D_{\delta_{2}}(u, b, \infty)$ exists because $D_{\delta_{2}}(u, b, \infty)$ is increasing in $u$ and bounded by $\alpha / \delta_{2}$.

Next, for any realisation of $\{S(t)\}_{t \geq 0}$, the event $\left\{U_{b}(s) \geq b\right.$ for all $\left.s \geq 0\right\}$ (for $\left\{U_{b}(t)\right\}_{t \geq 0}$ starting with initial surplus $u \geq b$ ) is equivalent to the event $\left\{\tau_{u-b, 0}=\infty\right\}$ (for $\left\{U_{0}(t)\right\}_{t \geq 0}$ starting with $u-b)$. Since $\lim _{u \rightarrow \infty} \psi(u-b ; 0)=0$, using the same arguments leading to (A.7) yields $\lim _{u \rightarrow \infty} 1_{\left\{\tau_{u-b, 0}=\infty\right\}}=1$ a.s. and hence $\lim _{u \rightarrow \infty} 1_{\left\{U_{b}(s) \geq b \text { for all } s \geq 0\right\}}=1$ a.s.. By consolidating these observations, it is found that

$$
\begin{aligned}
\lim _{u \rightarrow \infty} \varphi_{\delta_{23}, n, m}(u ; b) & =E\left[Z_{\delta_{3}}^{m}(\infty) \lim _{u \rightarrow \infty}\left(D_{\delta_{2}}^{n}(u, b, \infty) 1_{\left\{U_{b}(s) \geq b \text { for all } s \geq 0\right\}}\right)\right] \\
& =E\left[Z_{\delta_{3}}^{m}(\infty) \lim _{u \rightarrow \infty}\left(\left(\frac{\alpha}{\delta_{2}}\right)^{n} 1_{\left\{U_{b}(s) \geq b \text { for all } s \geq 0\right\}}\right)\right] \\
& =\left(\frac{\alpha}{\delta_{2}}\right)^{n} E\left[\left(\sum_{k=1}^{\infty} e^{-\delta_{3} T_{k}} f\left(Y_{k}\right)\right)^{m}\right]
\end{aligned}
$$

Note that we have also used the fact that $D_{\delta_{2}}(u, b, \infty)=\alpha \int_{0}^{\infty} e^{-\delta_{2} s} 1_{\left\{U_{b}(s) \geq b\right\}} d s=\alpha / \delta_{2}$ on the set $\left\{U_{b}(s) \geq b\right.$ for all $\left.s \geq 0\right\}$ as well as $Z_{\delta_{3}}(\infty)=\sum_{k=1}^{\infty} e^{-\delta_{3} T_{k}} f\left(Y_{k}\right)$ a.s. in the last two equalities. From the definition (2.11), the result (2.12) follows, from which it is clear that the right-hand side is finite if $\theta_{\delta_{3}, m}$ is finite (and from Remark 6 a sufficient condition for this is that the $m$ th moment of $f\left(Y_{1}\right)$ is finite). 
Proof of Lemma 3: Suppose that $w(\cdot, \cdot)$ is bounded by a constant $W$. Using the notations in the proof of Lemma 2, we have that, for $n, m \in \mathbb{N}$

$$
\begin{aligned}
\lim _{u \rightarrow \infty} \phi_{\delta_{123}, n, m}(u ; b)= & \lim _{u \rightarrow \infty} E\left[e^{-\delta_{1} \tau_{u, b}} D_{\delta_{2}}^{n}\left(u, b, \tau_{u, b}\right) Z_{\delta_{3}}^{m}\left(\tau_{u, b}\right) w\left(U_{b}\left(\tau_{u, b}^{-}\right), U_{b}\left(\tau_{u, b}\right)\right) 1_{\left\{\tau_{u, b}<\infty\right\}}\right] \\
& \leq W \lim _{u \rightarrow \infty} E\left[D_{\delta_{2}}^{n}\left(u, b, \tau_{u, b}\right) Z_{\delta_{3}}^{m}\left(\tau_{u, b}\right) 1_{\left\{\tau_{u, b}<\infty\right\}}\right] \\
& =W \lim _{u \rightarrow \infty}\left(E\left[D_{\delta_{2}}^{n}\left(u, b, \tau_{u, b}\right) Z_{\delta_{3}}^{m}\left(\tau_{u, b}\right)\right]-E\left[D_{\delta_{2}}^{n}\left(u, b, \tau_{u, b}\right) Z_{\delta_{3}}^{m}\left(\tau_{u, b}\right) 1_{\left\{\tau_{u, b}=\infty\right\}}\right]\right) \\
& =W\left(\lim _{u \rightarrow \infty} E\left[D_{\delta_{2}}^{n}\left(u, b, \tau_{u, b}\right) Z_{\delta_{3}}^{m}\left(\tau_{u, b}\right)\right]-\lim _{u \rightarrow \infty} \varphi_{\delta_{23}, n, m}(u ; b)\right)
\end{aligned}
$$

One can apply Monotone Convergence Theorem to the first limit to yield

$$
\lim _{u \rightarrow \infty} E\left[D_{\delta_{2}}^{n}\left(u, b, \tau_{u, b}\right) Z_{\delta_{3}}^{m}\left(\tau_{u, b}\right)\right]=E\left[\lim _{u \rightarrow \infty}\left(D_{\delta_{2}}^{n}\left(u, b, \tau_{u, b}\right) Z_{\delta_{3}}^{m}\left(\tau_{u, b}\right)\right)\right]=\lim _{u \rightarrow \infty} \varphi_{\delta_{23, n}, m}(u ; b)
$$

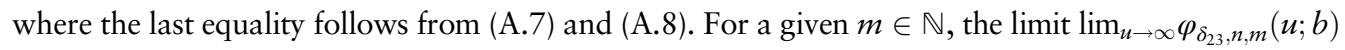
on the right-hand side is finite according to Lemma 2 since we assume that $\theta_{\delta_{3}, m}$ is finite. As both limits appearing in (A.10) are equal, one has that $\lim _{u \rightarrow \infty} \phi_{\delta_{123}, n, m}(u ; b) \leq 0$, which along with the nonnegativity of $\phi_{\delta_{123}, n, m}(u ; b)$ results in (2.13). (Note that we require both $E\left[D_{\delta_{2}}^{n}\left(u, b, \tau_{u, b}\right) Z_{\delta_{3}}^{m}\left(\tau_{u, b}\right)\right]$ and $E\left[D_{\delta_{2}}^{n}\left(u, b, \tau_{u, b}\right) Z_{\delta_{3}}^{m}\left(\tau_{u, b}\right) 1_{\left\{\tau_{u, b}=\infty\right\}}\right]$ to be finite in obtaining the equality (A.9). But this must be true as both expectations are increasing in $u$ and converge to a finite limit.)

Proof of Theorem 4: When $m=0$, the IDE (2.5) becomes, for $0<u<b$

$$
c_{1} \phi_{L, \delta_{12, n}}^{\prime}(u ; b)-\left(\lambda+\delta_{1}+n \delta_{2}\right) \phi_{L, \delta_{12}, n}(u ; b)+\lambda \int_{0}^{u} \phi_{L, \delta_{12}, n}(u-y ; b) p(y) d y=0
$$

which is structurally identical to e.g. Gerber et al. (2006, equation (2.12)). Therefore, the solution form (3.13) along with (3.16) is a direct consequence of their equations (7.3) and (7.8).

Next, (3.14), (3.15), and (3.17) can be proved by induction on $n \in \mathbb{N}^{+}$. To begin, we look at the case $n=1$. From (2.6), we have that, for $u>b$

$$
\begin{aligned}
& c_{2} \phi_{U, \delta_{12}, 1}^{\prime}(u ; b)-\left(\lambda+\delta_{1}+\delta_{2}\right) \phi_{U, \delta_{12}, 1}(u ; b)+\alpha \phi_{U, \delta_{1}}(u ; b)+\lambda \int_{0}^{u-b} \phi_{U, \delta_{12}, 1}(u-y ; b) p(y) d y \\
& \quad+\lambda \int_{u-b}^{u} \phi_{L, \delta_{12}, 1}(u-y ; b) p(y) d y=0
\end{aligned}
$$

Using the density (3.1) and (3.13) with $n=1$ leads the last integral in (A.11) to

$$
\lambda \int_{u-b}^{u} \phi_{L, \delta_{12}, 1}(u-y ; b) p(y) d y=\lambda \sum_{j=1}^{r+1} \sum_{k=1}^{r} \frac{A_{1,0,0, j} q_{k} \mu_{k}}{\mu_{k}+\rho_{1,0, j}}\left(e^{\left(\mu_{k}+\rho_{1,0, j}\right) b}-1\right) e^{-\mu_{k} u}
$$

Because $\left(d / d u+\mu_{k}\right) \int_{0}^{u-b} \phi_{U, \delta_{12}, 1}(u-y ; b) e^{-\mu_{k} y} d y=\phi_{U, \delta_{12}, 1}(u ; b)$, insertion of (3.1), (A.12), and $\phi_{U, \delta_{1}}(u ; b)$ given in Lemma 4 into (A.11) followed by application of the operator $\prod_{k=1}^{r}\left(d / d u+\mu_{k}\right)$ results in an $(r+1)$ th order differential equation with constant coefficients satisfied by $\phi_{U, \delta_{12}, 1}(\cdot ; b)$. Note that the non-homogeneous part of this differential equation involves the exponential 
terms $\left\{e^{\kappa_{0,0, j} u}\right\}_{j=1}^{r}$. Let $\left\{\kappa_{1,0, j}\right\}_{j=1}^{r+1}$ be the roots of the characteristic equation of the homogeneous part. Later we will see that $\left\{\kappa_{1,0, j}\right\}_{j=1}^{r+1}$ are really Lundberg's roots defined via (3.2). Then, we arrive at the solution form

$$
\phi_{U, \delta_{12}, 1}(u ; b)=\sum_{j=1}^{r} A_{1,0,0,0, j}^{*} e^{\kappa_{0,0, j} u}+\sum_{j=1}^{r+1} A_{1,0,1,0, j}^{*} e^{\kappa_{1,0, j} u}, \quad u>b
$$

where $A_{1,0, i, 0, j}^{*}$ 's are constants to be determined. Utilising (3.1), (3.8), and (A.13), we evaluate the first four terms in (A.11) as

$$
\begin{aligned}
& c_{2} \phi_{U, \delta_{12}, 1}^{\prime}(u ; b)-\left(\lambda+\delta_{1}+\delta_{2}\right) \phi_{U, \delta_{12,1}}(u ; b)+\alpha \phi_{U, \delta_{1}}(u ; b)+\lambda \int_{0}^{u-b} \phi_{U, \delta_{12}, 1}(u-y ; b) p(y) d y \\
& =c_{2}\left(\sum_{j=1}^{r} A_{1,0,0,0, j}^{*} \kappa_{0,0, j} e^{\kappa_{0,0, j} u}+\sum_{j=1}^{r+1} A_{1,0,1,0, j}^{*} \kappa_{1,0, j} e^{\kappa_{1,0, j} u}\right) \\
& -\left(\lambda+\delta_{1}+\delta_{2}\right)\left(\sum_{j=1}^{r} A_{1,0,0,0, j}^{*} e^{\kappa_{0,0, j} u}+\sum_{j=1}^{r+1} A_{1,0,1,0, j}^{*} e^{\kappa_{1,0, j} u}\right)+\alpha \sum_{j=1}^{r} A_{0,0,0,0, j}^{*} e^{\kappa_{0,0 j} u} \\
& +\lambda \sum_{j=1}^{r} \sum_{k=1}^{r} \frac{A_{1,0,0,0, j}^{*} q_{k} \mu_{k}}{\mu_{k}+\kappa_{0,0, j}}\left(e^{\kappa_{0,0, j} u}-e^{\left(\mu_{k}+\kappa_{0,0, j}\right) b} e^{-\mu_{k} u}\right)+\lambda \sum_{j=1}^{r+1} \sum_{k=1}^{r} \frac{A_{1,0,1,0, j}^{*} q_{k} \mu_{k}}{\mu_{k}+\kappa_{1,0, j}}\left(e^{\kappa_{1,0, j} u}-e^{\left(\mu_{k}+\kappa_{1,0, j}\right) b} e^{-\mu_{k} u}\right)
\end{aligned}
$$

As the sum of (A.12) and (A.14) is 0 for all $u>b$ due to (A.11), relationships among the unknown constants in (A.13) can be obtained by equating various exponential terms with 0 . First, examining the coefficients of $e^{\kappa_{1,0, j} u}$ asserts that $\left\{\kappa_{1,0, j}\right\}_{j=1}^{r+1}$ are the roots of (3.2) when $l=2, n=1$, and $m=0$. Since $\kappa_{1,0, r+1}>0$, application of the limiting condition (2.13) to (A.13) reveals that $A_{1,0,1,0, r+1}^{*}=0$ (see Remark 9), and therefore we have proved (3.14) when $n=1$. Next, from the coefficients of $e^{\kappa_{0,0 j} u}$, we get

$$
c_{2} A_{1,0,0,0, j}^{*} \kappa_{0,0, j}-\left(\lambda+\delta_{1}+\delta_{2}\right) A_{1,0,0,0, j}^{*}+\alpha A_{0,0,0,0, j}^{*}+\lambda \sum_{k=1}^{r} \frac{A_{1,0,0,0, j}^{*} q_{k} \mu_{k}}{\mu_{k}+\kappa_{0,0, j}}=0, \quad j=1,2, \ldots, r
$$

which leads to (3.15) when $n=1$ because each $\kappa_{0,0, j}$ satisfies (3.2) when $l=2$ and $n=m=0$. Lastly, the coefficients of $e^{-\mu_{\kappa} u}$ along with the use of $A_{1,0,1,0, r+1}^{*}=0$ imply

$$
-\lambda \sum_{i=0}^{1} \sum_{j=1}^{r} \frac{A_{1,0, i, 0, j}^{*} q_{k} \mu_{k}}{\mu_{k}+\kappa_{i, 0, j}} e^{\left(\mu_{k}+\kappa_{i, 0, j}\right) b}+\lambda \sum_{j=1}^{r+1} \frac{A_{1,0,0, j} q_{k} \mu_{k}}{\mu_{k}+\rho_{1,0, j}}\left(e^{\left(\mu_{k}+\rho_{1,0, j}\right) b}-1\right)=0, \quad k=1,2, \ldots, r
$$

One can use (3.16) when $n=1$ to simplify the above equation and observe that (3.17) holds true when $n=1$. Having shown that (3.14), (3.15), and (3.17) are true for $n=1$, mathematical induction can be applied to prove that they are also valid for all $n \in \mathbb{N}^{+}$. Since the induction step is almost identical to the above proof, the details are omitted here. Finally, (3.18) is a result of the continuity condition (2.7) and the solutions (3.13) and (3.14).

Proof of Theorem 5: It is instructive to note that the analysis of the IDE for the lower layer is identical to that in Theorem 4. Therefore, it is clear that (3.19) and (3.22) hold true. Moreover, once the solution (3.20) in the upper layer is proved (in what follows), (3.24) simply comes from the continuity condition (2.10). 
Like the proof of Theorem 4, we shall focus on proving (3.20), (3.21), and (3.23) for $n=1$, as the induction step is essentially the same as this first step. When $n=1$ and $m=0,(2.9)$ becomes, for $u>b$

$$
\begin{aligned}
& c_{2} \varphi_{U, \delta_{2}, 1}^{\prime}(u ; b)-\left(\lambda+\delta_{2}\right) \varphi_{U, \delta_{2}, 1}(u ; b)+\alpha \varphi_{U}(u ; b)+\lambda \int_{0}^{u-b} \varphi_{U, \delta_{2}, 1}(u-y ; b) p(y) d y \\
& \quad+\lambda \int_{u-b}^{u} \varphi_{L, \delta_{2}, 1}(u-y ; b) p(y) d y=0
\end{aligned}
$$

Although the above IDE looks identical to (A.11), it contains the starting point $\varphi_{U}(u ; b)$ (given in (3.12)) which involves an additional constant term compared to the starting point $\phi_{U, \delta_{1}}(u ; b)$ (given in (3.8)) of (A.11). See Remark 5. Similar to the derivations of Theorem 4, application of the operator $\prod_{k=1}^{r}\left(d / d u+\mu_{k}\right)$ leads (A.15) to an $(r+1)$ th order differential equation with constant coefficients as well as some non-homogeneous terms involving a constant and the exponential terms $\left\{e^{\kappa_{0,0, j}^{*} u}\right\}_{j=1}^{r}$. We shall see that the roots of the characteristic equation of the homogeneous part are the Lundberg's roots $\left\{\kappa_{1,0, j}^{*}\right\}_{j=1}^{r+1}$, and hence the general solution of $\varphi_{U, \delta_{2}, 1}(u ; b)$ is

$$
\varphi_{U, \delta_{2}, 1}(u ; b)=\sum_{j=1}^{r} C_{1,0,0,0, j}^{*} e^{\kappa_{0,0, j}^{*} u}+\sum_{j=1}^{r+1} C_{1,0,1,0, j}^{*} e^{\kappa_{1,0, j}^{*} u}+E_{1,0}, \quad u>b
$$

for some constants $C_{1,0,0,0, j}^{*}$ 's, $C_{1,0,1,0, j}^{*}$ 's, and $E_{1,0}$. Substitution of (3.1), (3.12), (3.19) with $n=1$, and (A.16) into the left-hand side of (A.15) followed by straightforward calculations yields

$$
\begin{aligned}
0= & c_{2}\left(\sum_{j=1}^{r} C_{1,0,0,0, j}^{*} \kappa_{0,0, j}^{*} e^{\kappa_{0,0, j}^{*} u}+\sum_{j=1}^{r+1} C_{1,0,1,0, j}^{*} \kappa_{1,0, j}^{*} e^{\kappa_{1,0, j}^{*} u}\right) \\
& -\left(\lambda+\delta_{2}\right)\left(\sum_{j=1}^{r} C_{1,0,0,0, j}^{*} e^{\kappa_{0,0, j}^{*} u}+\sum_{j=1}^{r+1} C_{1,0,1,0, j}^{*} e^{\kappa_{1,0, j}^{*} u}+E_{1,0}\right)+\alpha\left(\sum_{j=1}^{r} C_{0,0,0,0, j}^{*} e^{\kappa_{0,0, j}^{*} u}+1\right) \\
& +\lambda \sum_{j=1}^{r} \sum_{k=1}^{r} \frac{C_{1,0,0,0, j}^{*} q_{k} \mu_{k}}{\mu_{k}+\kappa_{0,0, j}^{*}}\left(e^{\kappa_{0,0, j}^{*} u}-e^{\left(\mu_{k}+\kappa_{0,0, j}^{*}\right)} e^{-\mu_{k} u}\right) \\
& +\lambda \sum_{j=1}^{r+1} \sum_{k=1}^{r} \frac{C_{1,0,1,0, j}^{*} q_{k} \mu_{k}}{\mu_{k}+\kappa_{1,0, j}^{*}}\left(e^{\kappa_{1,0, j}^{*} u}-e^{\left(\mu_{k}+\kappa_{1,0, j}^{*}\right) b} e^{-\mu_{k} u}\right)+\lambda E_{1,0} \sum_{k=1}^{r} q_{k}\left(1-e^{\mu_{k} b} e^{-\mu_{k} u}\right) \\
& +\lambda \sum_{j=1}^{r+1} \sum_{k=1}^{r} \frac{C_{1,0,0, j} q_{k} \mu_{k}}{\rho_{1,0, j}^{*}+\mu_{k}}\left(e^{\left(\mu_{k}+\rho_{1,0, j}^{*}\right) b}-1\right) e^{-\mu_{k} u}
\end{aligned}
$$

First, one confirms that $\left\{\kappa_{1,0, j}^{*}\right\}_{j=1}^{r+1}$ are the roots of (3.2) when $l=2, n=1$, and $m=\delta_{1}=0$ by equating the coefficients of $e^{\kappa_{1,0, j} u}$ with 0 . Noting that $\kappa_{1,0, r+1}^{*}>0$, the boundedness of $\varphi_{U, \delta_{2}, 1}(u ; b)$ as $u \rightarrow \infty$ according to Lemma 2 means that $C_{1,0,1,0, r+1}^{*}=0$. Second, the constant term implies $E_{1,0}=\alpha / \delta_{2}$, which must be the case because of $E_{1,0}=\lim _{u \rightarrow \infty} \varphi_{\delta_{23}, 1,0}(u)$ and Lemma 2 . Thus, the solution form (A.16) reduces to (3.20) when $n=1$. Finally, using the coefficients of $e^{\kappa_{0,0, j}^{*} u}$ and $e^{-\mu_{k} u}$, respectively, proves that (3.21) and (3.23) are true for $n=1$ in the same manner as (3.15) and (3.17) when $n=1$ are shown. The induction step of the proof of (3.20), (3.21), and (3.23) is omitted.

Proof of Theorem 6: We begin by recalling from Remark 4 that $\phi_{\delta_{13}, 1}(u ; b)=\phi_{L, \delta_{13}, 1}(u ; b)$ in the lower layer satisfies the same IDE as the counterpart under a dividend barrier strategy. As a result, 
some results in Cheung et al. (2015, theorem 6) are applicable. In particular, (3.25), (3.27), and (3.29) directly follow from equations (29)-(31) therein. Therefore, it is sufficient to look at the IDE in the upper layer. From (2.3), it is given by, for $u>b$

$$
\begin{aligned}
& c_{2} \phi_{U, \delta_{13}, 1}^{\prime}(u ; b)-\left(\lambda+\delta_{1}+\delta_{3}\right) \phi_{U, \delta_{13}, 1}(u ; b)+\lambda \int_{0}^{u-b} \phi_{U, \delta_{13}, 1}(u-y ; b) p(y) d y+\lambda \int_{u-b}^{u} \phi_{L, \delta_{13}, 1}(u-y ; b) p(y) d y \\
& +\lambda \int_{0}^{u-b} y \phi_{U, \delta_{1}}(u-y ; b) p(y) d y+\lambda \int_{u-b}^{u} y \phi_{L, \delta_{1}}(u-y ; b) p(y) d y+\lambda \int_{u}^{\infty} y w(y-u) p(y) d y=0
\end{aligned}
$$

Using (3.1), (3.7), (3.8), and (3.25), the last four integrals in (A.18) are evaluated as

$$
\lambda \int_{u-b}^{u} \phi_{L, \delta_{13}, 1}(u-y ; b) p(y) d y=\lambda \sum_{i=0}^{1} \sum_{j=1}^{r+1} \sum_{k=1}^{r} \frac{A_{0,1, i, j} q_{k} \mu_{k}}{\mu_{k}+\rho_{0, i, j}}\left(e^{\left(\mu_{k}+\rho_{0, i, j}\right) b}-1\right) e^{-\mu_{k} u}
$$

$$
\begin{aligned}
\lambda \int_{0}^{u-b} y \phi_{U, \delta_{1}}(u-y ; b) p(y) d y= & \lambda \sum_{j=1}^{r} \sum_{k=1}^{r} A_{0,0,0,0, j}^{*} q_{k} \mu_{k}\left[\frac{1}{\left(\mu_{k}+\kappa_{0,0, j}\right)^{2}} e^{\kappa_{0,0, j} u}-\frac{1}{\mu_{k}+\kappa_{0,0, j}} e^{\left(\mu_{k}+\kappa_{0,0, j}\right) b} u e^{-\mu_{k} u}\right. \\
& \left.+\left(\frac{b}{\mu_{k}+\kappa_{0,0, j}}-\frac{1}{\left(\mu_{k}+\kappa_{0,0, j}\right)^{2}}\right) e^{\left(\mu_{k}+\kappa_{0,0, j}\right) b} e^{-\mu_{k} u}\right]
\end{aligned}
$$

$$
\begin{aligned}
\lambda \int_{u-b}^{u} y \phi_{L, \delta_{1}}(u-y ; b) p(y) d y= & \lambda \sum_{j=1}^{r+1} \sum_{k=1}^{r} A_{0,0,0, j} q_{k} \mu_{k}\left\{\frac{1}{\mu_{k}+\rho_{0,0, j}}\left(e^{\left(\mu_{k}+\rho_{0,0, j}\right) b}-1\right) u e^{-\mu_{k} u}\right. \\
+ & {\left.\left[-\frac{b}{\mu_{k}+\rho_{0,0, j}} e^{\left(\mu_{k}+\rho_{0,0, j}\right) b}+\frac{1}{\left(\mu_{k}+\rho_{0,0, j}\right)^{2}}\left(e^{\left(\mu_{k}+\rho_{0,0, j}\right) b}-1\right)\right] e^{-\mu_{k} u}\right\}(\mathrm{A}, 2}
\end{aligned}
$$

and

$$
\lambda \int_{u}^{\infty} y w(y-u) p(y) d y=\lambda \sum_{k=1}^{r} q_{k} \mu_{k} \tilde{w}\left(\mu_{k}\right) u e^{-\mu_{k} u}+\lambda \sum_{k=1}^{r} q_{k} \mu_{k} \mathcal{T}_{\mu_{k}}^{2} w(0) e^{-\mu_{k} u}
$$

Applying the operator $\prod_{k=1}^{r}\left(d / d u+\mu_{k}\right)$ to $($ A.18) yields an $(r+1)$ th order differential equation with constant coefficients, and the non-homogeneous terms involve the exponential terms $\left\{e^{\kappa_{0,0}, j}\right\}_{j=1}^{r}$ and $\left\{e^{-\mu_{k} u}\right\}_{k=1}^{r}$. As the Lundberg's roots $\left\{\kappa_{0,1, j}\right\}_{j=1}^{r+1}$ will be shown to satisfy the characteristic equation of the homogeneous part, we arrive at the solution form

$$
\phi_{U, \delta_{13}, 1}(u ; b)=\sum_{j=1}^{r} A_{0,1,0,0, j}^{*} e^{\kappa_{0,0, j} u}+\sum_{j=1}^{r+1} A_{0,1,0,1, j}^{*} e^{\kappa_{0,1, j} u}+\sum_{k=1}^{r} B_{0,1,0, k}^{*} e^{-\mu_{k} u}, \quad u>b
$$

for some constants $A_{0,1,0, i, j}^{*}$ 's and $B_{0,1,0, k}^{*}$ 's. With (3.1) and (A.23), the first three terms in (A.18) are found to be

$$
c_{2} \phi_{U, \delta_{13}, 1}^{\prime}(u ; b)-\left(\lambda+\delta_{1}+\delta_{3}\right) \phi_{U, \delta_{13}, 1}(u ; b)+\lambda \int_{0}^{u-b} \phi_{U, \delta_{13}, 1}(u-y ; b) p(y) d y
$$




$$
\begin{aligned}
& =c_{2}\left(\sum_{j=1}^{r} A_{0,1,0,0, j}^{*} \kappa_{0,0, j} e^{\kappa_{0,0, j} u}+\sum_{j=1}^{r+1} A_{0,1,0,1, j}^{*} \kappa_{0,1, j} e^{\kappa_{0,1, j} u}-\sum_{k=1}^{r} B_{0,1,0, k}^{*} \mu_{k} e^{-\mu_{k} u}\right) \\
& -\left(\lambda+\delta_{1}+\delta_{3}\right)\left(\sum_{j=1}^{r} A_{0,1,0,0, j}^{*} e^{\kappa_{0,0, j} u}+\sum_{j=1}^{r+1} A_{0,1,0,1, j}^{*} e^{\kappa_{0,1, j} u}+\sum_{k=1}^{r} B_{0,1,0, k}^{*} e^{-\mu_{k} u}\right) \\
& +\lambda \sum_{j=1}^{r} \sum_{k=1}^{r} \frac{A_{0,1,0,0, j}^{*} q_{k} \mu_{k}}{\mu_{k}+\kappa_{0,0, j}}\left(e^{\kappa_{0,0, j} u}-e^{\left(\mu_{k}+\kappa_{0,0, j}\right) b} e^{-\mu_{k} u}\right)+\lambda \sum_{j=1}^{r+1} \sum_{k=1}^{r} \frac{A_{0,1,0,1, j}^{*} q_{k} \mu_{k}}{\mu_{k}+\kappa_{0,1, j}}\left(e^{\kappa_{0,1, j} u}-e^{\left(\mu_{k}+\kappa_{0,1, j}\right) b} e^{-\mu_{k} u}\right) \\
& +\lambda \sum_{k=1}^{r} \sum_{i=1, i \neq k}^{r} \frac{B_{0,1,0, i}^{*} q_{k} \mu_{k}}{\mu_{k}-\mu_{i}}\left(e^{-\mu_{i} u}-e^{\left(\mu_{k}-\mu_{i}\right) b} e^{-\mu_{k} u}\right)+\lambda \sum_{k=1}^{r} B_{0,1,0, k}^{*} q_{k} \mu_{k}(u-b) e^{-\mu_{k} u}
\end{aligned}
$$

Owing to (A.18), the sum of (A.19)-(A.22) and (A.24) is identical to 0. From the coefficients of $e^{\kappa_{0,1, j} u}$, we know that $\left\{\kappa_{0,1, j}\right\}_{j=1}^{r+1}$ are the roots of (3.2) when $l=2, n=0$, and $m=1$. As $\kappa_{0,1, r+1}>0$, the limiting condition (2.13) implies $A_{0,1,0,1, r+1}^{*}=0$. Next, comparing the coefficients of $u e^{-\mu_{k} u}$ leads to

$$
\begin{gathered}
\lambda B_{0,1,0, k}^{*} q_{k} \mu_{k}-\lambda \sum_{j=1}^{r} \frac{A_{0,0,0,0, j}^{*} q_{k} \mu_{k}}{\mu_{k}+\kappa_{0,0, j}} e^{\left(\mu_{k}+\kappa_{0,0, j}\right) b}+\lambda \sum_{j=1}^{r+1} \frac{A_{0,0,0, j} q_{k} \mu_{k}}{\mu_{k}+\rho_{0,0, j}}\left(e^{\left(\mu_{k}+\rho_{0,0, j}\right) b}-1\right)+\lambda q_{k} \mu_{k} \tilde{w}\left(\mu_{k}\right)=0, \\
k=1,2, \ldots, r
\end{gathered}
$$

Rearrangements give

$$
\begin{array}{r}
B_{0,1,0, k}^{*}=\left(\sum_{j=1}^{r+1} \frac{A_{0,0,0, j}}{\mu_{k}+\rho_{0,0, j}}-\tilde{w}\left(\mu_{k}\right)\right)+\left(\sum_{j=1}^{r} \frac{A_{0,0,0,0, j}^{*}}{\mu_{k}+\kappa_{0,0, j}} e^{\kappa_{0,0, j} b}-\sum_{j=1}^{r+1} \frac{A_{0,0,0, j}}{\mu_{k}+\rho_{0,0, j}} e^{\rho_{0,0, j} b}\right) e^{\mu_{k} b}=0, \\
k=1,2, \ldots, r
\end{array}
$$

thanks to (3.9) and (3.10). Hence, (A.23) reduces to (3.26). Utilising the coefficients of $e^{\kappa_{0,0, j} u}$, one has $c_{2} A_{0,1,0,0, j}^{*} \kappa_{0,0, j}-\left(\lambda+\delta_{1}+\delta_{3}\right) A_{0,1,0,0, j}^{*}+\lambda \sum_{k=1}^{r} \frac{A_{0,1,0,0, j}^{*} q_{k} \mu_{k}}{\mu_{k}+\kappa_{0,0, j}}+\lambda \sum_{k=1}^{r} \frac{A_{0,0,0,0, j}^{*} q_{k} \mu_{k}}{\left(\mu_{k}+\kappa_{0,0, j}\right)^{2}}=0, \quad j=1,2, \ldots, r$

which results in (3.28) since each $\kappa_{0,0, j}$ satisfies the Lundberg's equation (3.2) when $l=2$ and $n=m=0$. Lastly, equating the coefficients of $e^{-\mu_{k} u}$ results in

$$
\begin{aligned}
& -\lambda \sum_{i=0}^{1} \sum_{j=1}^{r} \frac{A_{0,1,0, i, j}^{*} q_{k} \mu_{k}}{\mu_{k}+\kappa_{0, i, j}} e^{\left(\mu_{k}+\kappa_{0, i, j}\right) b}+\lambda \sum_{i=0}^{1} \sum_{j=1}^{r+1} \frac{A_{0,1, i, j} q_{k} \mu_{k}}{\mu_{k}+\rho_{0, i, j}}\left(e^{\left(\mu_{k}+\rho_{0, i, j}\right) b}-1\right) \\
& +\lambda \sum_{j=1}^{r} A_{0,0,0,0, j}^{*} q_{k} \mu_{k}\left(\frac{b}{\mu_{k}+\kappa_{0,0, j}}-\frac{1}{\left(\mu_{k}+\kappa_{0,0, j}\right)^{2}}\right) e^{\left(\mu_{k}+\kappa_{0,0, j}\right) b} \\
& +\lambda \sum_{j=1}^{r+1} A_{0,0,0, j} q_{k} \mu_{k}\left[-\frac{b}{\mu_{k}+\rho_{0,0, j}} e^{\left(\mu_{k}+\rho_{0,0, j}\right) b}+\frac{1}{\left(\mu_{k}+\rho_{0,0, j}\right)^{2}}\left(e^{\left(\mu_{k}+\rho_{0,0, j}\right) b}-1\right)\right] \\
& +\lambda q_{k} \mu_{k} \mathcal{T}_{\mu_{k}}^{2} w(0)=0, \quad k=1,2, \ldots, r
\end{aligned}
$$

With the help of (3.10) and (3.29), simplifications of the above equation yield (3.30). The remaining formula (3.31) comes from the continuity condition (2.4). 